\title{
MATRIX ELEMENTS IN THE Sp(6) $\supset$ U(3) BRANCH OF THE S, D FERMION PAIR MODEL
}

\author{
K.T. HECHT \\ Physics Department, University of Michigan, Ann Arbor, MI 48109, USA \\ Jin-Quan CHEN \\ Department of Physics and Atmospheric Science, Drexel University, Philadelphia, PA 19104, USA \\ and \\ Department of Physics, Nanjing University, Nanjing, P.R. China \\ Received 16 November 1989 \\ (Revised 11 January 1990)
}

\begin{abstract}
General expressions are given for the matrix elements of single-nucleon creation and annihilation operators for one-body and pair operators coupled to arbitrary $i$-space spin, in the $\operatorname{Sp}(6) \supset U(3)$ basis of the S, D pair algebra. These should facilitate calculations for rotational nuclei in the fermion dynamic symmetry model.
\end{abstract}

\section{Introduction}

In recent years the Ginocchio S, D fermion pair algebra ${ }^{1}$ ) has been proposed as a fermion dynamic symmetry model ${ }^{2-5}$ ) which can serve as a microscopic model for collective excitations in nuclei throughout the periodic chart ${ }^{6-8}$ ). In this model the normal-parity single-particle states of the shell model are reclassified in terms of a pseudo-orbital angular momentum $(k)$ and pseudo spin $(i)$, with $\boldsymbol{k}+\boldsymbol{i}=\boldsymbol{j}$. The so-called $k$-active version of the model, with $k$ limited to $k=1$, leads to a $\operatorname{Sp}(6)$ symmetry with a $S p(6) \supset U(3)$ branch which can lead to strongly rotational spectra, particularly in the actinide region, where both the normal $(-)$ parity valence protons with $j=\frac{1}{2}, \frac{3}{2}, \frac{5}{2}, \frac{7}{2}, \frac{9}{2}$, (modeled by $k=1 ; i=\frac{1}{2}, \frac{7}{2}$ ), and the normal ( + ) parity neutrons with $j=\frac{1}{2}, \frac{3}{2}, \frac{5}{2}, \frac{7}{2}, \frac{9}{2}, \frac{11}{2}$; (with $k=1 ; i=\frac{3}{2}, \frac{9}{2}$ ) may lead to collective states of good $\mathrm{Sp}(6)$ symmetry. Very recently, it has also been shown that the new vector coherent state techniques ${ }^{9-11}$ ) are tailor-made to construct the matrix representations of all branches of the Ginocchio S, D pair algebra ${ }^{12-14}$ ). The matrix elements of the group generators are therefore known. For the $S p(6) \supset U(3)$ branch and states of low generalized S, D-pair seniority or "heritage", $u$, particularly for $u=0,1,2$, matrix elements of the group generators have been given essentially in analytic form ${ }^{14,15}$ ). This has made possible detailed studies of model hamiltonians built from the group

\footnotetext{
${ }^{1}$ Supported in part by the US National Science Foundation.
} 
generators ${ }^{16}$ ). It is one of the great advantages of the fermion dynamic symmetry model that, unlike the interacting boson model of Arima and Iachello, it is firmly based on the nuclear shell model. There is therefore no necessity to build model hamiltonians in terms of the group generators. The $\mathrm{Sp}(6) \supset \mathrm{U}(3)$ basis states can be used as a collective basis for the most general shell model interactions, and transition probabilities can be calculated in terms of the true electromagnetic moment operators, rather than model quadrupole moment operators similar to those used in the interacting boson model. For such calculations, however, it becomes necessary to evaluate $\mathrm{Sp}(6) \supset \mathrm{U}(3)$ matrix elements of operators lying outside the $\mathrm{Sp}(6)$ algebra, in particular operators coupled to a total $i$-spin other than zero.

Very recently, vector coherent state theory has been generalized to include the coherent state realizations of simple operators lying outside the group algebra ${ }^{17}$ ). It is the purpose of this contribution to apply this generalization of vector coherent state theory to evaluate the $\mathrm{Sp}(6) \supset \mathrm{U}(3)$ matrix elements of operators lying outside the $S p(6)$ algebra. In particular, matrix elements of single-nucleon creation and annihilation operators can lead to general cfp expressions. Matrix elements are also given for the most general one-body unit tensors, coupled to arbitrary $k$ and $i$-space angular momenta, together with the corresponding matrix elements of nucleon pair-creation and annihilation operators. These should facilitate calculations in the $\mathrm{Sp}(6) \supset \mathrm{U}(3)$ basis of the fermion dynamic symmetry model. Such matrix elements are needed, in particular, to calculate the single-particle energy contributions, single-particle transfer strengths, and $B(E 2)$ values as well as other electromagnetic transition probabilities. The fermion dynamic symmetry model assumes that the low-lying collective states of nuclei are dominated by states of low "heritage", $u$. Matrix elements of operators coupled to arbitrary $k$-space and $i$-space spherical tensor character should make it possible to test the goodness of this assumption. An assessment can now be made of the importance of higher heritage admixtures using realistic effective interactions.

The strategy for the evaluation of matrix elements is that common to all seniority schemes. The matrix elements for the $n$-particle system are converted to those of the $u$ - or $(u+1)$ - or $(u+2)$-particle system. For sufficiently low $u$, the latter are then reduced to simple standard shell-model calculations. Vector coherent state (VCS) theory is tailor-made for this purpose since the VCS realization of a general operator factors this operator into two parts in a coupled basis, an "intrinsic" operator acting only in the $\boldsymbol{u}$-particle subspace and a "collective" operator acting in the subspace of favored S, D-pair excitations, similar to the "spin" and "orbital" operators in an SLJ basis. Sect. 2 gives the needed VCS realization of the basic unit operators of this investigation as well as the $u$-space reduced matrix elements of the intrinsic operators introduced by this realization. These are summarized through a catalogue of intrinsic operator reduced matrix elements in table 3 . This catalogue converts the needed intrinsic operator matrix elements to the corresponding standard shell-model matrix elements connecting states of heritage, $u$, to states of $u^{\prime}=u, u \pm 1$, 
or $u \pm 2$. Very specific examples of these so-called starting matrix elements are given in an appendix. In sect. 3 the $k$-space $S U(3), i$-space $S U(2)$ reduced matrix elements of the single-nucleon creation and annihilation operators for the $n$-particle system are then reduced to those for the $u$-particle system by standard $S U(3), S U(2)$ recoupling techniques. Very specific results for $u$ and $u^{\prime} \leqslant 2$ are tabulated in terms of $\operatorname{Sp}(6) \supset \mathrm{U}(3)$ reduced Wigner coefficients in tables 5-11. Sect. 4 gives the reduction of the $n$-particle matrix elements for the nucleon pair-creation (annihilation) operators and the one-body unit tensors or multipole operators. The needed $n$ particle matrix elements are collected in a catalogue of general matrix elements in table 4. These matrix elements are expressed in terms of SU(3) recoupling coefficients which are readily available through the code of Draayer and Akiyama ${ }^{18}$ ) and the starting matrix elements illustrated by the simple examples of the appendix. In some simple special cases, of particular interest for fermion dynamic symmetry model calculations, the needed SU(3) coefficients can be given in analytic form making it possible to give simple analytic formulae for the needed matrix elements. This is illustrated with some examples in sect. 4.

\section{The vector coherent state realizations}

In the S, D fermon pair model the single-nucleon creation operators, $a_{j m}^{\dagger}$, for the normal-parity partners of a major oscillator shell are given in terms of pseudo angular momenta $k$ and $i$, with $k+i=j$ :

$$
a_{j m}^{\dagger}=\sum_{m_{k} m_{i}}\left\langle k m_{k} i m_{i} \mid j m\right\rangle b_{k m_{k} i m_{i}}^{\dagger} .
$$

For the $\operatorname{Sp}(6) k$-active version of the model, $k=1$, and it is useful to define cartesian components, $b_{\text {aim }}^{\dagger}$, where $a=x, y, z$, and

$$
b_{1 \pm 1 \text { i m }}^{\dagger}=\mp \sqrt{\frac{1}{2}}\left(b_{x i m_{i}}^{\dagger} \pm i b_{y i m_{i}}^{\dagger}\right), \quad b_{10 i m_{i}}^{\dagger}=b_{z i m_{i}}^{\dagger} .
$$

The $\operatorname{Sp}(6)$ algebra is then generated by the pair creation operators

$$
A_{a b}^{\dagger}=A_{b a}^{\dagger}=\sum_{i, m_{i}}(-1)^{i-m_{i}} b_{a i m_{i}}^{\dagger} b_{b i-m_{i}}^{\dagger},
$$

(with $a, b=x, y$, or $z$ ), the corresponding pair annihilation operators, $A_{a b}=\left(A_{a b}^{\dagger}\right)^{\dagger}$, and the one-body operators, $C_{a b}$, coupled to total $i$-spin of zero with a coherent sum over all $i$, [see eqs. (2)-(6) of ref. $\left.{ }^{14}\right)$ ]. The vector coherent state, (VCS), is built in terms of the six complex variables $z_{a b}\left(=z_{b a}\right)$

$$
|z\rangle=\exp \left(\frac{1}{2} \sum_{a b} z_{a b}^{*} A_{a b}^{\dagger}\right)|[\sigma] \alpha\rangle,
$$

where $[\sigma] \equiv\left[\sigma_{1} \sigma_{2} \sigma_{3}\right]$ is the "intrinsic" $\mathrm{U}(3)$ symmetry of the $u$ nucleons entirely free of the favored S, D pairs of the model, where the generalized seniority $u$ (or "heritage") is given by $u=\sigma_{1}+\sigma_{2}+\sigma_{3}$. In eq. (4), $\alpha$ stands for any set of convenient 
$\mathrm{U}(3)$ subgroup labels. The $\sigma_{i}$ also label the $\mathrm{Sp}(6)$ representations

$$
\left(\Omega_{1} \Omega_{2} \Omega_{3}\right)=\left(\frac{1}{3} \Omega-\sigma_{3}, \frac{1}{3} \Omega-\sigma_{2}, \frac{1}{3} \Omega-\sigma_{1}\right), \quad \text { with } \Omega=3 \sum_{i}\left(i+\frac{1}{2}\right)
$$

In the VCS method, state vectors are mapped into $z$-space functional realizatons

$$
|\Psi\rangle \rightarrow \Psi_{[\sigma] \alpha}(z)=\left\langle[\sigma] \alpha\left|\mathrm{e}^{z \cdot A}\right| \Psi\right\rangle, \quad \text { with }(z \cdot A)=\frac{1}{2} \sum_{a b} z_{a b} A_{a b},
$$

and operators $\boldsymbol{O}$ are mapped into their $z$-space realizations, $\Gamma(\boldsymbol{O})$,

$$
\begin{aligned}
\boldsymbol{O}|\Psi\rangle & \rightarrow\left\langle[\sigma] \alpha\left|\mathrm{e}^{z \cdot A} \boldsymbol{O} \mathrm{e}^{-z \cdot A} \mathrm{e}^{z \cdot A}\right| \Psi\right\rangle=\left\langle[\sigma] \alpha\left|\Gamma(\boldsymbol{O}) \mathrm{e}^{z \cdot A}\right| \Psi\right\rangle \\
& =\left\langle[\sigma] \alpha\left|\left\{\boldsymbol{O}+[z \cdot \boldsymbol{A}, \boldsymbol{O}]+\frac{1}{2}[[z \cdot \boldsymbol{A},[z \cdot \boldsymbol{A}, \boldsymbol{O}]]]+\cdots\right\} \mathrm{e}^{z \cdot A}\right| \Psi\right\rangle
\end{aligned}
$$

The $z$-space realizations of the $\operatorname{Sp}(6)$ generators are given in eq. (12) of ref. ${ }^{14}$ ). We now want to generalize the VCS method to include operators $\boldsymbol{O}$ outside the algebra, in particular the single-nucleon operators $b^{\dagger}, b$, and the one-body operators $\left[b^{\dagger} \times b\right]$, as well as the pair operators $\left[b^{\dagger} \times b^{\dagger}\right]$ and $[b \times b]$ where the latter are not restricted to $i$-space angular momenta of zero but are coupled to arbitrary $i$-space angular momenta. These operators can be organized into $\mathrm{Sp}(6) \supset \mathrm{U}(3)$ irreducible tensors $T_{[\omega] \alpha, I M,}^{\left(\Omega_{1} \Omega_{2} \Omega_{3}\right)}$ as shown in table 1 , where $[\omega] \equiv\left[\omega_{1} \omega_{2} \omega_{3}\right]$ is the $U(3)$ irreducible representation with subgroup labels $\alpha$. The single-nucleon $b^{\dagger}, b$ transform according to the 6-dimensional $\operatorname{Sp}(6)$ representation, $\left(\Omega_{1} \Omega_{2} \Omega_{3}\right)=(100)$, where for fixed $i, m_{i}$ the subgroup label $\alpha$ could take on the three values $\alpha \equiv a=x, y$, or $z$. Alternatively, $\alpha \equiv 1 m_{k}$ in a $\mathrm{U}(3) \supset \mathrm{SO}(3)$ basis, (see table 1 which exhibits both the $k$-space

\begin{tabular}{|c|c|}
\hline$\left(\omega_{0}\right)$ & $\left(\Omega_{1} \Omega_{2} \Omega_{3}\right)=(100)-$ operators \\
\hline$(10)$ & $b_{\alpha i m_{i}}^{\dagger}=\left(b^{\dagger}\right)_{\alpha, i m_{i}}^{(10)}$ \\
\hline$(01)$ & $-b_{\alpha i-m_{i}}(-1)^{i+m_{i}+x}=-(\tilde{b})_{\tilde{\alpha}, i m_{i}}^{(01)}$ \\
\hline$(\omega)$ & $\left(\Omega_{1} \Omega_{2} \Omega_{3}\right)=(200)$ - operators \\
\hline$(20)$ & $\sqrt{\frac{1}{2}} A^{\dagger}\left(i i^{\prime}\right)_{K M_{K}, I M_{l}}^{(20)}=\sqrt{\frac{1}{2}}\left[b_{i}^{\dagger} \times b_{i}^{\dagger}\right]_{K M_{K}, I M_{l}}^{(20)} \quad(K=0,2)$ \\
\hline$(00)$ & $-P\left(i i^{\prime}\right)_{K M_{K}, I M_{I}}^{(s)\left(\omega_{0}\right)}=-\frac{1}{2}\left\{\left[b_{i}^{\dagger} \times \tilde{b}_{i}\right]_{K M_{K}, I M_{i}}^{\left(\omega_{0}\right)}-(-1)^{i+i^{\prime}-1}\left[b_{i}^{\dagger} \times \tilde{b}_{i}\right]_{K M_{K}, I M_{i}}^{\left(\omega_{0}\right)}\right\}$ \\
\hline (11) & $K=0$ for $\left(\omega_{0}\right)=(00), \quad K=1,2$ for $\left(\omega_{0}\right)=(11)$ \\
\hline$(02)$ & $\sqrt{\frac{1}{2}} A\left(i i^{\prime}\right)_{K M_{K}, I M_{i}}^{(02)}=\sqrt{\frac{1}{2}}\left[\tilde{b}_{i} \times \tilde{b}_{i^{\prime}}\right]_{K M_{K}, I M_{i}}^{(02)} \quad(K=0,2)$ \\
\hline$(\omega)$ & $\left(\Omega_{1} \Omega_{2} \Omega_{3}\right)=(110)$ - operators \\
\hline$(01)$ & $\sqrt{\frac{1}{2}} A^{\dagger}\left(i i^{\prime}\right)_{K M_{K}, I M_{l}}^{(01)}=\sqrt{\frac{1}{2}}\left[b_{i}^{\dagger} \times b_{i}^{\dagger}\right]_{K M_{K}, I M_{l}}^{(01)} \quad(K=1)$ \\
\hline (11) & $-P\left(i i^{\prime}\right)_{K M_{K}, I M_{l}}^{(\mathrm{a})(11)}=-\frac{1}{2}\left\{\left[b_{i}^{\dagger} \times \tilde{b}_{i^{\prime}}\right]_{K M_{K}, I M_{I}}^{(11)}+(-1)^{i+i^{\prime}-I}\left[b_{i^{\prime}}^{\dagger} \times \tilde{b}_{i}\right]_{K M_{K}, I M_{l}}^{(11)}\right\}$ \\
\hline (10) & $\sqrt{\frac{1}{2}} A\left(i i^{\prime}\right)_{K M_{K}, I M_{i}}^{(10)}=\sqrt{\frac{1}{2}}\left[\tilde{b}_{i} \times \tilde{b}_{i^{\prime}}\right]_{K M_{K}, I M_{i}}^{(10)} \quad(K=1)$ \\
\hline
\end{tabular}
$\mathrm{SU}(3) \supset \mathrm{SO}(3)$ and the $i$-space $\mathrm{SU}(2)$ irreducible tensor character of $b^{\dagger}$ and $b$ ).

TABLE 1

The basic tensors $T^{\left(\Omega_{1} \Omega_{2} \Omega_{3}\right)}$ 
Throughout the paper the SU(3) labels $\left(\lambda_{\omega} \mu_{\omega}\right) \equiv\left(\omega_{1}-\omega_{2}, \omega_{2}-\omega_{3}\right)$ will often be abbreviated by $(\omega)$, in round parentheses, whereas the U(3) labels $\left[\omega_{1} \omega_{2} \omega_{3}\right]$ will be abbreviated by $[\omega]$, in square brackets. Note also that a $k$-space SU(3), $i$-space $\mathrm{SU}(2)$ double tensor has the conjugation property

$$
\left(T_{\alpha M_{l}}^{(\omega) I}\right)^{\dagger}=T_{\tilde{\alpha}-M_{l}}^{(\tilde{\omega}) I}(-1)^{\chi(\omega, \alpha)}(-1)^{I-M_{l}},
$$

where $(\tilde{\omega})=\left(\mu_{\omega} \lambda_{\omega}\right), \tilde{\alpha}=K,-M_{K}$. The SU(3) conjugation phase factor, $\chi(\omega, \alpha)$, is somewhat dependent on phase conventions and on the specific choice of subgroup label $\alpha$. However, this phase factor drops out of all final expressions for $\mathrm{SU}(3)$ reduced matrix elements.

The one-body and pair creation (annihilation) operators with antisymmetric $i$-space coupling belong to the 21 -dimensional $S p(6)$ representation (200), while those with symmetric $i$-space coupling belong to the 14-dimensional $S p(6)$ representation (110). The coupled pair creation operators are defined through

$$
\begin{aligned}
A^{\dagger}\left(i i^{\prime}\right)_{K M_{K} I M_{I}}^{\left(\omega_{p}\right)} & =\left[b_{i}^{\dagger} \times b_{i^{\prime}}^{\dagger}\right]_{K M_{K} I M_{l}}^{\left(\omega_{p}\right)}=\left[b_{i}^{\dagger} \times b_{i^{\prime}}^{\dagger}\right]_{K M_{K} I M_{l}}\left\langle(10) 1 ;(10) 1||\left(\omega_{p}\right) K\right\rangle, \\
{\left[b_{i}^{\dagger} \times b_{i^{\prime}}^{\dagger}\right]_{K M_{K} I M_{I}} } & =\sum_{m_{k} m_{k^{\prime}}} \sum_{m_{i} m_{i^{\prime}}}\left\langle 1 m_{k} 1 m_{k^{\prime}} \mid K M_{K}\right\rangle\left\langle i m_{i} i^{\prime} m_{i}^{\prime} \mid I M_{I}\right\rangle b_{1 m_{k} i m i}^{\dagger} b_{1 m_{k^{\prime}} i^{\prime} m_{i^{\prime}}}^{\dagger},
\end{aligned}
$$

where $K=0,2$ for the $\mathrm{SU}(3)$ symmetric representation $\left(\omega_{p}\right)=\left(\omega_{\mathrm{s}}\right)=(20)$, while $K=1$ for the $\operatorname{SU}(3)$ antisymmetric representation $\left(\omega_{p}\right)=\left(\omega_{\mathrm{a}}\right)=(01)$. Note the identity

$$
\left[b_{i}^{\dagger} \times b_{i^{\prime}}^{\dagger}\right]_{K M_{K}, I M_{I}} \equiv \frac{1}{2}\left\{\left[b_{i}^{\dagger} \times b_{i^{\prime}}^{\dagger}\right]_{K M_{K}, I M_{i}} \mp\left[b_{i^{\prime}}^{\dagger} \times b_{i}^{\dagger}\right]_{K M_{K}, I M_{l}}(-1)^{i+i^{\prime}-I}\right\},
$$

for $K=0,2$ (or $K=1$ ) upper (lower) signs, respectively; and note that $I$ is automatically restricted to even (odd) integer values for $K=0,2$ (or $K=1$ ) in the special case $i=i^{\prime}$. Note also that the SU(3) $\supset \mathrm{SO}(3)$ Wigner coefficient, the double-barred coefficient of eq. (9a) has absolute value of unity for the trivial couplings of eq. (9a). The coupled pair annihilation operators are defined similarly by

$$
\begin{aligned}
A\left(i i^{\prime}\right)_{K M_{K}, I M_{I}}^{\left(\tilde{\omega}_{p}\right)} & =\left[\tilde{b}_{i} \times \tilde{b}_{i^{\prime}}\right]_{K M_{K}, I M_{I}}^{\left(\tilde{\omega}_{p}\right)} \\
& =\left(A^{\dagger}\left(i i^{\prime}\right)_{K-M_{K}, I-M_{I}}^{\left(\omega_{p}\right)}\right)^{\dagger}(-1)^{1+\hat{\omega}_{p}+K-M_{K}+I-M_{I}} .
\end{aligned}
$$

The SU(3) phase factor, designated by $\hat{\omega}$, arises in many conjugation and SU(3)coupling reordering transformations. It is defined through

$$
(-1)^{\hat{\omega}} \equiv(-1)^{\lambda_{\omega}+\mu_{\omega}}=(-1)^{\omega_{1}-\omega_{3}} .
$$

Finally, the one-body unit tensors or multipole operators are defined through

$$
P\left(i i^{\prime}\right)_{K M_{K}, I M_{I}}^{(p)\left(\omega_{0}\right)}=\frac{1}{2}\left\{\left[b_{i}^{\dagger} \times \tilde{b}_{i^{\prime}}\right]_{K M_{K}, I M}^{\left(\omega_{0}\right)} \mp(-1)^{i+i^{\prime}-I}\left[b_{i^{\prime}}^{\dagger} \times \tilde{b}_{i}\right]_{K M_{K}, I M_{l}}^{\left(\omega_{0}\right)}\right\}
$$

where the operators with $(p)=(s)$, (upper sign) and $\left(\omega_{0}\right)=(00)$ or $(11)$, belong to the family of $\operatorname{Sp}(6)$ (200)-operators, whereas those with $(p)=(\mathrm{a})$, (lower sign), and $\left(\omega_{0}\right)=(11)$ only belong to the $\operatorname{Sp}(6)$ (110)-operators. The symbol s(a) denotes that they are symmetric (antisymmetric) in the $k$-space. 
The $z$-space realizations of the above operators can now be constructed by the applications of eq. (7). The $z$-space realizations $\Gamma(\boldsymbol{O})$ in eq. (7) consist of two types of operators, $z$-space polynomials or "collective operators", and fermion operators such as $b^{\dagger}, b, A\left(i i^{\prime}\right)$, etc. The latter commute with the $z$-space polynomials and are to be interpreted through their left actions on the vacuum or "intrinsic" states with quantum numbers $[\sigma] \alpha$. Following the standard VCS notation these "intrinsic" operators are denoted by $b^{\dagger}, b, \mathbb{A}(i i)$, etc. Since intrinsic operators such as $b^{\dagger}, b, A(i i), \ldots$ are defined through their left actions on intrinsic states, they are to be interpreted as follows: They must always be commuted through to the left in a matrix element so that they can act on the intrinsic state. Eq. (7) gives the following $\Gamma(\boldsymbol{O})$ :

$$
\begin{aligned}
& \Gamma\left(\tilde{b}_{a i m_{i}}\right)=\tilde{b}_{a i m_{i}}, \\
& \Gamma\left(b_{a i m_{i}}^{\dagger}\right)=b_{a i m_{i}}^{\dagger}-2\left[\tilde{\mathrm{b}}_{i}^{(01)} \times Z^{(20)}(z)\right]_{a}^{(10)}, \\
& \Gamma\left(A\left(i i^{\prime}\right)_{\alpha, I M_{l}}^{\left(\tilde{\omega}_{p}\right)}\right)=\mathbb{A}\left(i i^{\prime}\right)_{\alpha, I M,}^{\left(\tilde{\omega}_{p}\right)}, \\
& \Gamma\left(P\left(i i^{\prime}\right)_{\alpha, I M_{l}}^{(p)\left(\omega_{0}\right)}\right)=\mathbb{P}\left(i i^{\prime}\right)_{\alpha, I M_{l}}^{(p)\left(\omega_{0}\right)}+c_{\left(\omega_{0}\right)}^{(p)}\left[\mathbb{A}\left(i i^{\prime}\right)_{I}^{\left(\tilde{\omega}_{p}\right)} \times Z^{(20)}(z)\right]_{\alpha, I M_{l}}^{\left(\omega_{0}\right)}, \\
& \Gamma\left(A^{\dagger}\left(i i^{\prime}\right)_{\alpha, I M_{l}}^{\left(\omega_{p}\right)}\right)=\mathbb{A}^{\dagger}\left(i i^{\prime}\right)_{\alpha, I M,}^{\left(\omega_{p}\right)}+\sum_{\left(\omega_{0}\right)} f_{\left(\omega_{0}\right)}^{(p)}\left[\mathbb{P}\left(i i^{\prime}\right) l^{(p)\left(\omega_{0}\right)} \times Z^{(20)}(z)\right]_{\alpha, I M M_{l}}^{\left(\omega_{p}\right)} \\
& +\sum_{(n)} g_{(n)}^{(p)}\left[\left.A\left(i i^{\prime}\right)\right|^{\left(\tilde{\omega}_{p}\right)} \times Z^{(n)}(z)\right]_{\alpha, I M,}^{\left(\omega_{p}\right)} \\
& +\delta_{\left(\omega_{p}\right)(20)} \delta_{i i} \delta_{I 0} \sqrt{2 i+1} Z_{\alpha}^{(20)}(z),
\end{aligned}
$$

where the coefficients are given in table 2.

In eqs. (12a)-(12e), $\alpha$ is any convenient SU(3) subgroup label, such as the appropriate $K M_{K}$. As in eqs. (9) and (11) square brackets denote both SU(3) coupling and $i$-space angular momentum coupling. In eqs. (12a)-(12e) however, a

\begin{tabular}{|c|c|c|c|c|c|c|c|c|c|}
\hline \multirow{2}{*}{$(p)$} & \multirow{2}{*}{$\left(\omega_{p}\right)$} & \multirow{2}{*}{$\left(\tilde{\omega}_{p}\right)$} & \multirow{2}{*}{$\left(\omega_{0}\right)$} & \multicolumn{2}{|c|}{$c_{\left(\omega_{0}\right)}^{(p)}$} & \multicolumn{2}{|c|}{$f_{\left(\omega_{0}\right)}^{(p)}$} & \multicolumn{2}{|c|}{$g_{\left(\begin{array}{l}(p) \\
(n)\end{array}\right)}$} \\
\hline & & & & $c_{(00)}^{(p)}$ & $c_{(11)}^{(p)}$ & $f_{(00)}^{(p)}$ & $f_{(11)}^{(p)}$ & $g_{(40)}^{(p)}$ & $g_{(02)}^{(\rho)}$ \\
\hline (s) & $(20)$ & $(02)$ & $(00),(11)$ & -2 & $-\sqrt{\frac{5}{2}}$ & $-\sqrt{\frac{8}{3}}$ & $-\sqrt{\frac{40}{3}}$ & $2 \sqrt{5}$ & $-\sqrt{2}$ \\
\hline (a) & $(01)$ & (10) & (11) & & $+\sqrt{\frac{3}{2}}$ & & +4 & & $2 \sqrt{3}$ \\
\hline
\end{tabular}
right to left coupling order is used. This simplifies phases in the VCS construction and will be used henceforth. [Note, however, that the coupled operators, such as $\left[b_{i}^{\dagger} \times b_{i^{\prime}}^{\dagger}\right]$, are defined in terms of their standard left to right coupling order, see eq. (9a).] The $z$-space polynomials are the polynomials used to construct the orthonor-

TABLE 2

Coefficients used in the equations for the $z$-space realizations $\Gamma(O)$ of the intrinsic states 
mal Bargmann state vectors

$$
\left.\left.\left[Z^{\left[n_{1} n_{2} n_{3}\right]}(z) \times \mid\left[\sigma_{1} \sigma_{2} \sigma_{3}\right]\right)_{\alpha}^{\left[\omega_{1} \omega_{2} \omega_{3}\right] \rho} \equiv \mid[[n] \times[\sigma]][\omega] \rho, \alpha\right) \equiv \mid \phi_{[n] \rho}\right),
$$

where the polynomials of degree $n_{1}+n_{2}+n_{3}$, with $n_{i}$ all even integers, carry the "collective" U(3) quantum numbers $\left[n_{1} n_{2} n_{3}\right]$ which are coupled with the "intrinsic" $\left[\sigma_{1} \sigma_{2} \sigma_{3}\right]$, (right to left coupling order), to resultant $\mathrm{U}(3)$ states $\left[\omega_{1} \omega_{2} \omega_{3}\right]$ with outer multiplicity label $\rho$, when needed. In eqs. $(12 \mathrm{a})-(12 \mathrm{e})$ the $Z^{(n)}(z)$ have been labeled by their equivalent $\mathrm{SU}(3)$ quantum numbers $(n) \equiv\left(\lambda_{n} \mu_{n}\right)=\left(n_{1}-n_{2}, n_{2}-n_{3}\right)$. For their full construction, including their normalization, see e.g. p. 81 of ref. $\left.{ }^{11}\right)$.

In taking matrix elements it will be useful to indicate specifically whether matrix elements are to be calculated through their $z$-space integrations or in standard Hilbert space form. For this reason, the $z$-space state vector of eq. (13) has been written with a round parenthesis, $\mid \ldots$. . The corresponding state vector, written in standard Hilbert space notation with angular carets, $|\ldots\rangle$,

$$
\left[Z^{\left[n_{1} n_{2} n_{3}\right]}\left(\boldsymbol{A}^{\dagger}\right) \times\left|\left[\sigma_{1} \sigma_{2} \sigma_{3}\right]\right\rangle\right]_{\alpha}^{\left[\omega_{1} \omega_{2} \omega_{3}\right] \rho} \equiv|[[n] \times[\sigma]][\omega] \rho, \alpha\rangle \equiv\left|\phi_{[n] \rho}\right\rangle,
$$

is constructed through the successive action of the fermion operators $\boldsymbol{A}^{\dagger}$ on the vacuum or "intrinsic" state with particle number, $u$. In eq. (14) the operator $A_{a b}^{\dagger}$ has replaced the $z_{a b}$ in the polynomial $Z^{[n]}$. The states of eq. (13) form an orthonormal set in $z$-space.

$$
\left(\phi_{\left[n^{\prime}\right] \rho^{\prime}} \mid \phi_{[n] \rho}\right)=\delta_{[n]\left[n^{\prime}\right]} \delta_{\rho \rho^{\prime}} .
$$

The states of eq. (14), however, form a nonorthonormal set, including redundant states for some $[\sigma][\omega]$ combinations. The overlap of the states (14) are given by the $\left(K K^{\dagger}\right)$ matrix which is central to VCS theory

$$
\left\langle\phi_{\left[n^{\prime}\right] \rho} \mid \phi_{[n] \rho}\right\rangle=\left(K K^{\dagger}\right)_{\left[n^{\prime}\right] \rho^{\prime},[n] \rho} .
$$

Note that the hermitian matrix $\left(K K^{\dagger}\right)$ was chosen to be real in all earlier VCS applications, see e.g. ref. $\left.{ }^{14}\right)$ where the $\left(K K^{\dagger}\right)$ are real symmetric matrices. In the VCS technique, $K$ is also interpreted as the operator which converts the nonunitary realization, $\Gamma(\boldsymbol{O})$, of an operator $\boldsymbol{O}$ into a unitary realization, $\gamma(\boldsymbol{O})$,

$$
\gamma(\boldsymbol{O})=K^{-1} \Gamma(\boldsymbol{O}) K
$$

To define $K^{-1}$ it is necessary to convert the ( $K K^{\dagger}$ ) matrices to diagonal form via the unitary matrix, $U$

$$
U\left(K K^{\dagger}\right) U^{\dagger}=\lambda \text {, }
$$

with $\lambda_{\nu \nu^{\prime}}=\delta_{\nu \nu^{\prime}} \lambda_{\nu}$. Note that zero eigenvalues of $\left(K K^{\dagger}\right)$ immediately signal the presence of Pauli-forbidden states. The Pauli-allowed states can be designated by the new quantum number $\nu=1,2, \ldots$ corresponding to the states with nonzero eigenvalues $\lambda_{\nu}$. (This quantum number was generally labeled by $i=1,2, \ldots$ in earlier VCS references. The notation has been changed to avoid confusion with the angular 
momentum quantum number i.) Eq. (18) leads to

$$
(K)_{[n] \rho, \nu}=\left(U^{\dagger}\right)_{[n] \rho, \nu} \sqrt{\lambda_{\nu}} \text {. }
$$

For Pauli-allowed states, with $\lambda_{\nu} \neq 0$,

$$
\left(K^{-1}\right)_{\nu,[n] \rho}=\frac{1}{\sqrt{\lambda_{\nu}}} U_{\nu,[n] \rho} .
$$

The states with the new quantum number $\nu$, corresponding to nonzero eigenvalues $\lambda_{\nu}$, form the orthonormal set

$$
|[\sigma][\omega] \nu, \alpha\rangle=\sum_{[n] \rho}\left(K^{-1}\right)_{\nu,[n] \rho}^{*}\left[Z^{\left[n_{1} n_{2} n_{3}\right]}\left(A^{\dagger}\right) \times\left|\left[\sigma_{1} \sigma_{2} \sigma_{3}\right]\right\rangle\right]_{\alpha}^{\left[\omega_{1} \omega_{2} \omega_{3}\right] \rho},
$$

where the orthonormality follows from eqs. (16), (19) and (20). It may also be useful to define a new orthonormal set through a linear combination of the $z$-space set of eq. (13), through

$$
\left.\mid[\sigma][\omega] \nu, \alpha)=\sum_{[n] \rho} \mid \phi_{[n] \rho}\right)\left(U^{\dagger}\right)_{[n] \rho, \nu} .
$$

Note that the operators $K$ or $K^{-1}$ acting on the members of this set are given through the eigenvalues, $\lambda_{\nu}$,

$$
\begin{aligned}
K \mid[\sigma][\omega] \nu, \alpha) & \left.=\sqrt{\lambda_{\nu}} \mid[\sigma][\omega] \nu, \alpha\right) \\
\left([\sigma][\omega] \nu, \alpha \mid K^{-1}\right. & =\left([\sigma][\omega] \nu, \alpha \mid \frac{1}{{\sqrt{\lambda_{\nu}}}_{\nu}} .\right.
\end{aligned}
$$

The matrix element of an operator $\boldsymbol{O}$ in the orthonormal basis $|[\sigma][\omega] \nu, \alpha\rangle$ can then be transcribed to the $z$-space matrix element if we use the unitary realization of the operator, $\gamma(\boldsymbol{O})$, and the orthonormal $z$-space states $\mid[\sigma][\omega] \nu, \alpha)$. The reduced matrix element relation is

$$
\begin{aligned}
\left\langle\left[\sigma^{\prime}\right]\left[\omega^{\prime}\right] \nu^{\prime} ; I^{\prime}\left\|\boldsymbol{O}^{\left[\omega_{0}\right] I} \mathrm{o}\right\|[\sigma][\omega] \nu ; I\right\rangle_{\rho_{0}} \\
=\left(\left[\sigma^{\prime}\right]\left[\omega^{\prime}\right] \nu^{\prime} ; I^{\prime}\left\|\gamma(\boldsymbol{O})^{\left[\omega_{0}\right] I_{0}}\right\|[\sigma][\omega] \nu ; I\right)_{\rho_{0}} \\
=\sum_{[n] \rho\left[n^{\prime}\right] \rho^{\prime}}\left(K^{-1}\left(\left[\sigma^{\prime}\right]\left[\omega^{\prime}\right]\right)\right)_{\nu^{\prime},\left[n^{\prime}\right] \rho^{\prime}} \\
\quad \times\left(\left[\left[n^{\prime}\right] \times\left[\sigma^{\prime}\right]\right]\left[\omega^{\prime}\right] \rho^{\prime} ; I^{\prime}\left\|\Gamma(\boldsymbol{O})^{\left[\omega_{0}\right] I_{0}}\right\|[[n] \times[\sigma]][\omega] \rho ; I\right)_{\rho_{0}} K([\sigma][\omega])_{[n] \rho, \nu},
\end{aligned}
$$

where we have used eqs. (17), (22), and (23), and where the dependence on $[\sigma]$ and $[\omega]$ is shown explicitly in the $K$ matrix elements. In eq. (24) a triple bar denotes the fact that the reduced matrix element is reduced with respect to both the $k$-space SU(3)-coupling and the $i$-space angular momentum coupling. Such reduced matrix elements are defined without $\left[2 I^{\prime}+1\right]^{-1 / 2}$ and SU(3) dimensional factors.

$$
\begin{aligned}
& \left\langle\left[\sigma^{\prime}\right]\left[\omega^{\prime}\right] \nu^{\prime} ; I^{\prime}\left\|T^{\left[\omega_{0}\right] I_{0}}\right\|[\sigma][\omega] \nu ; I\right\rangle_{\rho_{0}} \\
& \quad=\left\langle\left[\sigma^{\prime}\right]\left[\omega^{\prime}\right] \nu^{\prime} \alpha^{\prime} ; I^{\prime} M_{I}^{\prime}\right|\left[T^{\left[\omega_{0}\right] I_{0}} \times|[\sigma][\omega] \nu ; I\rangle\right]_{\alpha^{\prime} ; M_{i}^{\prime}}^{\left[\omega^{\prime}\right] \rho_{\rho^{\prime}}},
\end{aligned}
$$


where the square bracket denotes both the $U(3)$ coupling $[\omega] \times\left[\omega_{0}\right] \rightarrow\left[\omega^{\prime}\right]$ and the $i$-space angular momentum coupling $I \times I_{0} \rightarrow I^{\prime}$. The multiplicity label $\rho_{0}$ is rarely needed. The special case $\left(\omega_{0}\right)=(11),\left(\omega^{\prime}\right)=(\omega)$, is one of the few cases treated in this investigation in which the coupling $(\omega) \times(11)$ leads to a two-fold SU(3) multiplicity. In such a case the full matrix element will involve a sum over $\rho_{0}$

$$
\begin{aligned}
\left\langle\left[\sigma^{\prime}\right]\left[\omega^{\prime}\right] \nu^{\prime} \alpha_{\omega^{\prime}} ; I^{\prime} M_{I}^{\prime}\left|O_{\alpha_{0} M_{l_{0}}}^{\left[\omega_{0}\right] I_{0}}\right|[\sigma][\omega] \nu \alpha_{\omega} ; I M_{I}\right\rangle \\
=\sum_{\rho_{0}}\left\langle\left[\sigma^{\prime}\right]\left[\omega^{\prime}\right] \nu^{\prime} ; I^{\prime}\left\|\boldsymbol{O}^{\left[\omega_{0}\right] I_{0}}\right\|[\sigma][\omega] \nu ; I\right\rangle_{\rho_{0}} \\
\quad \times\left\langle[\omega] \alpha_{\omega} ;\left[\omega_{0}\right] \alpha_{0} \mid\left[\omega^{\prime}\right] \alpha_{\omega^{\prime}}\right\rangle_{\rho_{0}}\left\langle I M_{I} I_{0} M_{I_{0}} \mid I^{\prime} M_{I}^{\prime}\right\rangle .
\end{aligned}
$$

In the applications to the fermion dynamic symmetry model it will be important to choose the SU(3) subgroup labels, $\alpha_{\omega}, \alpha_{0}, \alpha_{\omega^{\prime}}$, in an $\mathrm{SU}(3) \supset \mathrm{SO}(3)$ basis including the $k$-space angular momentum quantum numbers $K M_{K}$, so that the full $\mathrm{SU}(3)$ Wigner coefficient will factor into a product of an ordinary $k$-space angular momentum Wigner coefficient and an $S U(3) \supset S O(3)$ reduced Wigner coefficient. The latter can be obtained in an orthonormalized basis from the code of Draayer and Akiyama ${ }^{18}$ ).

Since eq. (24) will be used repeatedly to convert a $z$-space matrix element into a standard Hilbert space matrix element or vice versa, it will be termed the master equation. Note again that round parentheses, $\mid \ldots)$, are used to signify $z$-space integrations, whereas carets, $|\ldots\rangle$, signify standard Hilbert space matrix elements.

When $[n]=\left[n^{\prime}\right]=[0]$, the master eq. (24) collapses to

$$
\left(\left[\sigma^{\prime}\right] ; I^{\prime}\left\|\Gamma(\boldsymbol{O})^{\left[\omega_{0}\right] I_{0}}\right\|[\sigma] ; I\right)=\left\langle\left[\sigma^{\prime}\right] ; I^{\prime}\left\|\boldsymbol{O}^{\left[\omega_{0}\right] I_{0}}\right\|[\sigma] ; I\right\rangle,
$$

where we have used the fact that $K$ and $K^{-1}$ are simple unit operators when acting on the "intrinsic" states $[\sigma]$ or $\left[\sigma^{\prime}\right]$.

Eq. (24) is given for the most general case, including all SU(3) multiplicity labels. Since the $[\sigma]$ 's for low S, D-pair seniorities are such that the products $[n] \times[\sigma]$ are generally free of multiplicities ${ }^{14}$ ), the labels $\rho$ and $\rho^{\prime}$ are generally not needed. Matrix elements of $b^{\dagger}$ and $b$, with $\left(\omega_{0}\right)=(10)$ and $(01)$, are also free of the label $\rho_{0}$. Since the method of calculation will be illustrated in detail through the operators $b^{\dagger}, b$, all multiplicity labels $\rho$ will be omitted in the remainder of this section and in sect. 3 where it will be assumed that all SU(3) couplings are free of multiplicities. In sect. 4 , however, where some multiplicity labels come into play, all formulae are displayed with their full multiplicity labeling.

Since the intrinsic operators, such as $b^{\dagger}, b, \mathbb{A}, \mathbb{P}, \ldots$ in their left action on the intrinsic or vacuum states in general change the $S p(6)$ irreducible representation, the first step in any calculation involves the evaluation of the reduced matrix elements of such operators. Since the operator $b^{\dagger}$ acting to the left on a state with $\left[\sigma^{\prime}\right], u^{\prime}=$ $\sigma_{1}^{\prime}+\sigma_{2}^{\prime}+\sigma_{3}^{\prime}=u-1$, must lower the seniority to $u^{\prime}-1=u-2$, we have the obvious result

$$
u^{\prime}=u-1: \quad\left(\left[\sigma^{\prime}\right] ; I^{\prime}\left\|b_{i}^{\dagger}\right\|[\sigma] u ; I\right)=0
$$


Note that $[\sigma]$ specifies both the $U(3)$ representation of the intrinsic state and the $\mathrm{Sp}(6)$ representation via eq. (5).

The reduced matrix element of the intrinsic operator $b_{i}$, on the other hand, will be different from zero in this case. It can be related to the corresponding standard Hilbert space matrix element. With $u^{\prime}=u-1$ the left action of $b_{i}$ will convert the intrinsic state $\left[\sigma^{\prime}\right]$ to an intrinsic state $[\sigma]$ with heritage $u$. Using $\Gamma\left(\tilde{b}_{i}\right)=\tilde{b}_{i}$, eq. (27) yields

$$
u^{\prime}=u-1: \quad\left(\left[\sigma^{\prime}\right] ; I^{\prime}\left\|\tilde{b}_{i}\right\|[\sigma] ; I\right)=\left\langle\left[\sigma^{\prime}\right] ; I^{\prime}\left\|\tilde{b}_{i}\right\|[\sigma] ; I\right\rangle
$$

On the other hand, with $u^{\prime}=u+1$, the left action of $b_{i}$ on an intrinsic state will now have to convert this state to one with particle number $n=u+2$ which is no longer an intrinsic state of the $\operatorname{Sp}(6)$ representation specified by the intrinsic $[\sigma]$ but instead corresponds to a state created from the intrinsic $[\sigma]$ by the $\operatorname{Sp}(6)$ excitation operator $A^{\dagger[2]}$. The normalized $z$-space realization of this state is given by

$$
\left.\left.\mathbb{|}[[2] \times[\sigma]][\bar{\sigma}] \alpha_{\bar{\sigma}}\right)=\left[Z^{[2]}(z) \times \mid[\sigma]\right)\right]_{\alpha_{\bar{\sigma}}}^{[\bar{\sigma}]} .
$$

The corresponding normalized state in standard Hilbert space is

$$
\left|[\sigma][\bar{\sigma}] \nu=[2], \alpha_{\tilde{\sigma}}\right\rangle=\frac{1}{K([\sigma][\bar{\sigma}])_{[2][2]}}\left[A^{\dagger[2]} \times|[\sigma]\rangle\right]_{\alpha_{\bar{\sigma}}}^{[\bar{\sigma}]} .
$$

For the first symplectic excitation the $K$-matrix is a $1 \times 1$ matrix so that the label $\nu$ can be identified with $\nu \equiv[n]=[2]$ and $K^{-1}$ serves as a simple normalization factor. Using the master equation (24), together with eq. (12a)

$$
\begin{aligned}
\left(\left[\sigma^{\prime}\right] ;\right. & \left.I^{\prime}\left\|K^{-1} \Gamma\left(\tilde{b}_{i}\right) K\right\|[[2] \times[\sigma]][\bar{\sigma}] ; I\right) \\
\quad= & K([\sigma][\bar{\sigma}])_{[2][2]}\left(\left[\sigma^{\prime}\right] ; I^{\prime}\left\|\tilde{b}_{i}\right\|[[2] \times[\sigma]][\bar{\sigma}] ; I\right) \\
\quad= & \left\langle\left[\sigma^{\prime}\right] ; I^{\prime}\left\|\tilde{b}_{i}\right\|[\sigma][\bar{\sigma}] \nu=[2] ; I\right\rangle \\
& =K^{-1}([\sigma][\bar{\sigma}])_{[2][2]}\left\langle\left[\sigma^{\prime}\right] ; I^{\prime}\left\|\tilde{b}_{i}\right\|\left[A^{\dagger[2]} \times[\sigma]\right][\bar{\sigma}] ; I\right\rangle,
\end{aligned}
$$

leading to

$$
\begin{aligned}
& \left(\left[\sigma^{\prime}\right] ; I^{\prime}\left\|\tilde{\mathbb{b}}_{i}^{[11]}\right\|[[2] \times[\sigma]][\bar{\sigma}] ; I\right) \\
& \quad=\frac{1}{K^{2}([\sigma][\bar{\sigma}])_{[2][2]}}\left\langle\left[\sigma^{\prime}\right] ; I^{\prime}\left\|\tilde{b}_{i}^{[11]}\right\|\left[A^{\dagger[2]} \times[\sigma]\right][\bar{\sigma}] ; I\right\rangle .
\end{aligned}
$$

The right-hand side can be simplified by using the SU(3) recoupling relation for SU(3)-tensors $T^{[\omega]}$

$$
\begin{aligned}
& \left\langle\left[\sigma^{\prime}\right]\left\|T^{[\omega]}\right\|\left[T^{\left[\omega^{\prime}\right]} \times[\sigma]\right][\bar{\sigma}]\right\rangle \\
& \quad=\sum_{\left[\omega_{0}\right]} U\left([\sigma]\left[\omega^{\prime}\right]\left[\sigma^{\prime}\right][\omega] ;[\bar{\sigma}]\left[\omega_{0}\right]\right)\left\langle\left[\sigma^{\prime}\right]\left\|\left[T^{[\omega]} \times T^{\left[\omega^{\prime}\right]}\right]^{\left[\omega_{0}\right]}\right\|[\sigma]\right\rangle,
\end{aligned}
$$


with inverse

$$
\begin{aligned}
& \left\langle\left[\sigma^{\prime}\right]\left\|\left[T^{[\omega]} \times T^{\left[\omega^{\prime}\right]}\right]^{\left[\omega_{0}\right]}\right\|[\sigma]\right\rangle \\
& \quad=\sum_{[\tilde{\sigma}]} U\left([\sigma]\left[\omega^{\prime}\right]\left[\sigma^{\prime}\right][\omega] ;[\bar{\sigma}]\left[\omega_{0}\right]\right)\left\langle\left[\sigma^{\prime}\right]\left\|T^{[\omega]}\right\|\left[T^{\left[\omega^{\prime}\right]} \times[\sigma]\right][\bar{\sigma}]\right\rangle .
\end{aligned}
$$

With (33a), eq. (32) can be rewritten

$$
\begin{aligned}
\left(\left[\sigma^{\prime}\right] ; I^{\prime}\left\|\tilde{b}_{i}^{[11]}\right\|[[2] \times[\sigma]][\bar{\sigma}] ; I\right)= & \frac{1}{K^{2}([\sigma][\bar{\sigma}])_{[2][2]}} \sum_{\left[\omega_{0}\right]} U\left([\sigma][2]\left[\sigma^{\prime}\right][11] ;[\bar{\sigma}]\left[\omega_{0}\right]\right) \\
& \times\left\langle\left[\sigma^{\prime}\right] ; I^{\prime}\left\|\left[\tilde{b}_{i}^{[11]}, A^{\dagger[2]}\right]^{\left[\omega_{0}\right]}\right\|[\sigma] ; I\right\rangle
\end{aligned}
$$

where the $U(3)$ coupled operator $\left[\tilde{b}_{i}^{[11]} \times A^{+[2]}\right]^{\left[\omega_{0}\right]}$ has been converted to a $\mathrm{U}(3)$ coupled commutator by using the fact that $A^{\dagger}$ annihilates the intrinsic state $\left\langle\left[\sigma^{\prime}\right] \alpha^{\prime}\right|$ in its left action on such a state. The U(3)-coupled commutator is defined by

$$
\begin{aligned}
{\left[\tilde{b}_{i m_{i}}^{[11]}, A^{\dagger[2]}\right]_{\alpha}^{\left[\omega_{0}\right]} } & =\sum_{\alpha_{11}, \alpha_{2}}\left\langle[2] \alpha_{2} ;[11] \alpha_{11} \mid\left[\omega_{0}\right] \alpha\right\rangle\left[\tilde{b}_{\alpha_{11} i m_{i}}^{[11]}, A_{\alpha_{2}}^{\dagger[2]}\right] \\
& =2 \delta_{\left[\omega_{0}\right][1]} b_{\alpha i m_{i}}^{\dagger[1]}
\end{aligned}
$$

where straightforward anticommutation relations of the fermion operators have been used to evaluate the right-hand side of eq. (35). This leads to the result

$$
\text { for } \begin{aligned}
u^{\prime}=u+1: \quad\left(\left[\sigma^{\prime}\right] ;\right. & I^{\prime}\left\|\tilde{b}_{i}\right\|[[[2] \times[\sigma]][\bar{\sigma}] ; I) \\
= & \frac{2}{K^{2}([\sigma][\bar{\sigma}])_{[2][2]}} U\left([\sigma][2]\left[\sigma^{\prime}\right][11] ;[\bar{\sigma}][1]\right) \\
& \times\left\langle\left[\sigma^{\prime}\right] ; I^{\prime}\left\|b_{i}^{\dagger}\right\|[\sigma] ; I\right\rangle .
\end{aligned}
$$

The required matrix element resulting from the left action of the intrinsic operator $b_{i}$ on the intrinsic state $\left[\sigma^{\prime}\right]$ has thus been expressed in terms of a simple standard fermion matrix element of the operator $b_{i}^{\dagger}$, connecting the $u$-particle state to a $u^{\prime}=(u+1)$-particle state. Examples of these simple "starting matrix elements" are given in the appendix.

In similar fashion eq. (27), together with (12b), leads to

$$
\begin{aligned}
\left\langle\left[\sigma^{\prime}\right] ; I^{\prime}\left\|b_{i}^{\dagger}\right\|[\sigma] ; I\right\rangle= & \left(\left[\sigma^{\prime}\right] ; I^{\prime}\left\|\Gamma\left(b_{i}^{\dagger}\right)\right\|[\sigma] ; I\right) \\
= & \left(\left[\sigma^{\prime}\right] ; I^{\prime}\left\|b_{i}^{\dagger}\right\|[\sigma] ; I\right) \\
& -2\left(\left[\sigma^{\prime}\right] ; I^{\prime}\left\|\left[\tilde{b}_{i}^{[11]} \times Z^{[2]}(z)\right]^{[1]}\right\|[\sigma] ; I\right) .
\end{aligned}
$$


Eq. (33b) then gives

$$
\begin{aligned}
& \left(\left[\sigma^{\prime}\right] ; I^{\prime}\left\|\left[\tilde{\mathbf{b}}_{i}^{[11]} \times Z^{[2]}\right]^{[1]}\right\|[\sigma] ; I\right) \\
& \quad=\sum_{[\tilde{\sigma}]} U\left([\sigma][2]\left[\sigma^{\prime}\right][11] ;[\bar{\sigma}][1]\right)\left(\left[\sigma^{\prime}\right] ; I^{\prime}\left\|\tilde{\mathfrak{b}}_{i}^{[11]}\right\|[[2] \times[\sigma]][\bar{\sigma}] ; I\right) .
\end{aligned}
$$

The matrix element on the right-hand side is given by eq. (36) so that

$$
\text { for } \begin{aligned}
u^{\prime}=u+1: \quad\left(\left[\sigma^{\prime}\right] ; I^{\prime}\left\|b_{i}^{\dagger}\right\|[\sigma] ; I\right)= & \left\{1+4 \sum_{[\bar{\sigma}]} \frac{U^{2}\left([\sigma][2]\left[\sigma^{\prime}\right][11] ;[\bar{\sigma}][1]\right)}{K^{2}([\sigma][\bar{\sigma}])_{[2][2]}}\right\} \\
& \times\left\langle\left[\sigma^{\prime}\right] ; I^{\prime}\left\|b_{i}^{\dagger}\right\|[\sigma] ; I\right\rangle,
\end{aligned}
$$

with a nonzero value only for $u^{\prime}=u+1$.

Eqs. (36) and (39) can be evaluated in terms of simple U(3) or equivalent $\mathrm{SU}(3)$ Racah coefficients and the $K^{2}$ matrix elements for the first symplectic excitation with $[n]=[2]=[200]$. These are simple $1 \times 1$ submatrices with values which follow at once from eq. (24) of ref. ${ }^{14}$ ).

$$
\begin{aligned}
K^{2}([\sigma][\bar{\sigma}])_{[2][2]} & =\left(\frac{2}{3} \Omega-2 \sigma_{1}\right) & & \text { for }[\bar{\sigma}]=\left[\sigma_{1}+2, \sigma_{2}, \sigma_{3}\right], \\
& =\left(\frac{2}{3} \Omega-2 \sigma_{2}+2\right) & & \text { for }[\bar{\sigma}]=\left[\sigma_{1}, \sigma_{2}+2, \sigma_{3}\right], \\
& =\left(\frac{2}{3} \Omega-2 \sigma_{3}+4\right) & & \text { for }[\bar{\sigma}]=\left[\sigma_{1}, \sigma_{2}, \sigma_{3}+2\right], \\
& =\left(\frac{2}{3} \Omega-\sigma_{1}-\sigma_{2}+2\right) & & \text { for }[\bar{\sigma}]=\left[\sigma_{1}+1, \sigma_{2}+1, \sigma_{3}\right], \\
& =\left(\frac{2}{3} \Omega-\sigma_{1}-\sigma_{3}+3\right) & & \text { for }[\bar{\sigma}]=\left[\sigma_{1}+1, \sigma_{2}, \sigma_{3}+1\right], \\
& =\left(\frac{2}{3} \Omega-\sigma_{2}-\sigma_{3}+4\right) & & \text { for }[\bar{\sigma}]=\left[\sigma_{1}, \sigma_{2}+1, \sigma_{3}+1\right] .
\end{aligned}
$$

The techniques illustrated in connection with the results given by eqs. (36) and (39) can be used to evaluate the required reduced matrix elements of intrinsic operators such as $\mathbb{A}\left(i i^{\prime}\right)$ or $\mathbb{P}\left(i i^{\prime}\right)$. These are collected in table 3 . The results of table 3 are valid for the most general representations $[\sigma]$ and $\left[\sigma^{\prime}\right]$ and therefore include some SU(3) multiplicity labels $\rho_{0}$. In most cases of practical interest, however, these labels are not needed. For low values of $u$ (or $u^{\prime}$ ), in particular for $u\left(u^{\prime}\right) \leqslant 2$, the couplings $[\sigma] \times\left[\omega_{0}\right] \rightarrow\left[\sigma^{\prime}\right]$ are entirely multiplicity-free so that the labels $\rho_{0}$ and the $\rho_{0}$-sums can be omitted.

\section{Matrix elements of single-nucleon operators}

The single-nucleon creation (annihilation) operators can connect states of generalized S, D-pair seniority $u=\sigma_{1}+\sigma_{2}+\sigma_{3}$ to states with $u^{\prime}=u \pm 1$. VCS theory leads to particularly simple formulae for the case $u^{\prime}=u-1$. These will therefore be evaluated in detail. Formulae for the case $u^{\prime}=u+1$ are then obtained most simply via hermitian conjugation. 
TABLE 3

Catalogue of intrinsic operator reduced matrix elements

1. For $b_{i}^{\dagger}$ see eqs. (28) and (39)

2. For $\tilde{b}_{i}$ see eqs. (29) and (36)

3. $u^{\prime}=u-2$ :

$\left(\left[\sigma^{\prime}\right] ; I^{\prime}\left\|\mathbb{A}\left(i i^{\prime}\right)_{l_{p}}^{\left(\tilde{\omega}_{p}\right)}\right\|[\sigma] ; I\right)=\left\langle\left[\sigma^{\prime}\right] ; I^{\prime}\left\|A\left(i i^{\prime}\right)_{I_{p}}^{\left(\tilde{\omega}_{p}\right)}\right\|[\sigma \sigma] ; I\right\rangle$

4. $u^{\prime}=u$ :

$\left(\left[\sigma^{\prime}\right] ; I^{\prime}\left\|\mathbb{A}\left(i i^{\prime}\right) i_{p}^{\left(\tilde{\omega}_{p}^{\prime}\right)}\right\|[[2] \times[\sigma]][\bar{\sigma}] ; I\right)$

$$
\begin{aligned}
= & \frac{1}{K^{2}([\sigma][\tilde{\sigma}])_{[2][2]}}\left\{\sum_{\left[\omega_{0}\right] \rho_{0}} h_{\left(\omega_{0}\right)}^{(p)} U\left([\sigma][2]\left[\sigma^{\prime}\right]\left[\tilde{\omega}_{p}\right] ;[\bar{\sigma}]_{--} ;\left[\omega_{0}\right]_{-} \rho_{0}\right)\right. \\
& \times\left\langle\left[\sigma^{\prime}\right] ; I^{\prime}\left\|P\left(i i^{\prime}\right)(p)\left(\omega_{0}^{\prime}\right)\right\|[\sigma] ; I\right\rangle_{\rho_{0}} \\
& \left.-2 \sqrt{3(2 i+1)} U([\sigma][2][\sigma][22] ;[\bar{\sigma}][0]) \delta_{i i^{\prime}}, \delta_{I_{p} 0} \delta_{\left[\tilde{\omega}_{p}\right][22]} \delta_{l l^{\prime}} \delta_{[\sigma]\left[\sigma^{\prime}\right]}\right\}
\end{aligned}
$$

5. $u^{\prime}=u-2$ :

$$
\left.\left(\left[\sigma^{\prime}\right] ; I^{\prime} \| \mathbb{P}\left(i i^{\prime}\right)\right]_{p}^{(p)\left(\omega_{0}\right)} \|[\sigma \sigma] ; I\right)_{\rho_{0}}=0
$$

6. $u^{\prime}=u$ :

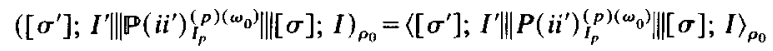

$$
\begin{aligned}
& -c_{\left(\omega_{0}\right)}^{(p)} \sum_{[\bar{\sigma}]} U\left([\sigma][2]\left[\sigma^{\prime}\right]\left[\tilde{\omega}_{p}\right] ;[\bar{\sigma}]_{--;}\left[\omega_{0}\right]_{-} \rho_{0}\right) \\
& \times\left(\left[\sigma^{\prime}\right] ; I^{\prime}\left\|\mathbb{A}\left(i i^{\prime}\right)_{i_{p}}^{\left(\tilde{\omega}_{p}\right)}\right\|[[2] \times[\sigma]][\bar{\sigma}] ; I\right)
\end{aligned}
$$

$$
\begin{aligned}
& \text { where } \left.\quad c_{(00)}^{(\mathrm{s})}=-2, c_{(11)}^{(\mathrm{s})}=-\sqrt{\frac{5}{2}} ; \quad c_{(11)}^{(\mathrm{a})}=+\sqrt{\frac{3}{2}} \quad \text { [see eqs. }(12 \mathrm{a})-(12 \mathrm{e})\right] \\
& h_{(00)}^{(\mathrm{s})}=4, h_{(11)}^{(\mathrm{s})}=\sqrt{10} ; \quad h_{(11)}^{(\mathrm{a})}=-\sqrt{6} \\
& \left(\tilde{\omega}_{s}\right)=(02) ; \quad\left(\tilde{\omega}_{a}\right)=(10) \\
& U\left([\sigma][2]\left[\sigma^{\prime}\right][22] ;[\bar{\sigma}][0]\right)=\delta_{[\sigma]\left[\sigma^{\prime}\right]}(-1)^{\hat{\sigma}+\hat{\sigma}} \sqrt{\operatorname{dim}[\bar{\sigma}] / 6 \operatorname{dim}[\sigma]}
\end{aligned}
$$

$$
(-1)^{\hat{\sigma}} \equiv(-1)^{\lambda_{\sigma}+\mu_{r \sigma}}=(-1)^{\sigma_{1}-\sigma_{3}}
$$

By applying the master eq. (24) to the single-nucleon creation operator and using the $z$-space realization $\Gamma\left(b^{\dagger}\right)$ of eq. $(12 \mathrm{~b})$, we obtain

$$
\begin{aligned}
\left\langle\left[\sigma^{\prime}\right]\left[\omega^{\prime}\right] \nu^{\prime} ; I^{\prime}\left\|b_{i}^{\dagger}\right\|[\sigma][\omega] \nu ; I\right\rangle \\
=\sum_{[n]\left[n^{\prime}\right]} K^{-1}\left(\left[\sigma^{\prime}\right]\left[\omega^{\prime}\right]\right)_{\nu^{\prime}\left[n^{\prime}\right]} K([\sigma][\omega])_{[n] \nu} \\
\quad \times\left\{\left(\left[\left[n^{\prime}\right] \times\left[\sigma^{\prime}\right]\right]\left[\omega^{\prime}\right] ; I^{\prime}\left\|\mathbb{L}_{i}^{\dagger[1]}\right\|[[[n] \times[\sigma]][\omega] ; I)\right.\right. \\
\left.\quad-2\left(\left[\left[n^{\prime}\right] \times\left[\sigma^{\prime}\right]\right]\left[\omega^{\prime}\right] ; I^{\prime}\left\|\left[Z^{[2]}(z) \times \tilde{b}_{i}^{[1]}\right]^{[1]}\right\|[[n] \times[\sigma]][\omega] ; I\right)\right\} .
\end{aligned}
$$

For the case $u^{\prime}=u-1$, the matrix element of the first term on the right is zero via eq. (28). The second term involves the matrix element of the intrinsic operator $b_{i}$ coupled to a $z$-space operator in a $U(3)$-coupled basis in which the state vectors involve the coupling of the intrinsic $[\sigma]$ to the $z$-space $[n]$. Note that the product 
$\left[\tilde{b}_{i}^{[11]} \times Z^{[2]}\right]^{[1]}$ is invariant under a change of coupling order and has been reordered in eq. (41) so that the coupling order of the operator is the same as that of the state vectors. For the case $u^{\prime}=u-1$ the left action of $b_{i}$ on the intrinsic state $\left[\sigma^{\prime}\right]$ connects this $\left[\sigma^{\prime}\right]$ to a purely intrinsic state $[\sigma]$ on the right via eq. (29). Straightforward recoupling techniques therefore convert this matrix element to the pure intrinsic space reduced matrix element of $b_{i}$ and the $z$-spaced reduced matrix element of $Z^{[2]}=\boldsymbol{z}$ to yield

$$
\begin{aligned}
\left\langle\left[\sigma^{\prime}\right]\left[\omega^{\prime}\right] \nu^{\prime} ;\right. & \left.I^{\prime}\left\|b_{i}^{\dagger}\right\|[\sigma][\omega] \nu ; I\right\rangle \\
= & -2 \sum_{\left[n^{\prime}\left[n^{\prime}\right]\right.} K^{-1}\left(\left[\sigma^{\prime}\right]\left[\omega^{\prime}\right]\right)_{\nu^{\prime}\left(n^{\prime}\right]} K([\sigma][\omega])_{[n] \nu}\left[\begin{array}{ccc}
{[\sigma]} & {[n]} & {[\omega]} \\
{[11]} & {[2]} & {[1]} \\
{\left[\sigma^{\prime}\right]} & {\left[n^{\prime}\right]} & {\left[\omega^{\prime}\right]}
\end{array}\right] \\
& \times\left(\left[n^{\prime}\right]\|z\|[n]\right)\left\langle\left[\sigma^{\prime}\right] ; I^{\prime}\left\|\tilde{b}_{i}\right\|[\sigma] ; I\right\rangle,
\end{aligned}
$$

where the [ ] symbol is a $\mathrm{U}(3)$ or equivalent $\mathrm{SU}(3)$ 9-j type symbol in unitary form. For simplicity it is assumed that $[\sigma]$ and $\left[\sigma^{\prime}\right]$ belong to the simple cases of ref. ${ }^{14}$ ) for which the $\mathrm{U}(3)$ products $[\sigma] \times[n] \rightarrow[\omega]$ are free of multiplicity. For the reduced matrix element of $z$ in the collective space characterized by $[n]$ and $\left[n^{\prime}\right]$; see e.g., p. 84 of ref. $\left.{ }^{11}\right)$. The intrinsic space reduced matrix element of $b_{i}$ has been converted to a matrix element of $b_{i}$ via eq. (29). For states of low generalized seniority, $u$, the reduced matrix elements of $b_{i}$ are easily evaluated by standard shell-model techniques. Examples of these starting matrix elements are given in the appendix.

For the case $u^{\prime}=u-1$ the matrix element of the single nucleon annihilation operator $b_{i}$ follows in similar fashion. In this case $\Gamma\left(b_{i}\right)$ is the pure intrinsic operator, $\mathbf{b}_{i}$, so that the recoupling coefficient in the final matrix element is a U-coefficient or SU(3) Racah coefficient in unitary form. The matrix element is listed as entry 2 in table 4 which gives a catalogue of all the reduced matrix elements evaluated in

TABLE 4

Catalogue for the matrix elements $\left.\left(\left[\left[n^{\prime}\right] \times\left[\sigma^{\prime}\right]\right]\left[\omega^{\prime}\right] \rho^{\prime} ; I^{\prime}\|\Gamma(O)\|[[n] \times[\sigma]] \omega\right] \rho ; I\right)_{\rho_{0}} \equiv M$

\begin{aligned} &$\left\langle\left[\sigma^{\prime}\right]\left[\omega^{\prime}\right] \nu^{\prime} ; I^{\prime}\|\boldsymbol{O}\|[\sigma][\omega] \nu ; I\right\rangle_{\rho_{0}}= \sum_{\left[n^{\prime}\right] \rho^{\prime}[[n] \rho} K^{-1}\left(\left[\sigma^{\prime}\right]\left[\omega^{\prime}\right]\right)_{\nu^{\prime},\left[n^{\prime}\right] \rho^{\prime}} K([\sigma][\omega])_{[n] \rho, \nu} \\ & \times\left(\left[\left[n^{\prime}\right] \times\left[\sigma^{\prime}\right]\right]\left[\omega^{\prime}\right] \rho^{\prime} ; I^{\prime}\|\Gamma(\boldsymbol{O})\|[[n] \times[\sigma]][\omega] \rho ; I\right)_{\rho_{0}} \\ &$\hline\end{aligned}

1. $O=b_{i}^{\dagger} ; \quad u^{\prime}=u-1$

2. $O=\tilde{b}_{i} ; \quad u^{\prime}=u-1$

$$
M=-2\left[\begin{array}{cccc}
{[\sigma]} & {[n]} & {[\omega]} & \rho \\
{[11]} & {[2]} & {[1]} & - \\
{\left[\sigma^{\prime}\right]} & {\left[n^{\prime}\right]} & {\left[\omega^{\prime}\right]} & \rho^{\prime} \\
- & - & - &
\end{array}\right]\left(\left[n^{\prime}\right]\|z\|[n]\right)\left\langle\left[\sigma^{\prime}\right] ; I^{\prime}\left\|\tilde{b}_{i}\right\|[\sigma] ; I\right\rangle
$$

$$
M=(-1)^{\hat{\sigma}-\hat{\sigma}^{\prime}+\hat{\omega}-\hat{\omega}^{\prime}} \delta_{[n]\left[n^{\prime}\right]} U\left([11][\sigma]\left[\omega^{\prime}\right][n] ;\left[\sigma^{\prime}\right] . \rho^{\prime} ;[\omega] \rho_{-}\right\rangle\left\langle\left[\sigma^{\prime}\right] ; I^{\prime}\left\|\tilde{b}_{i}\right\|[\sigma] ; I\right\rangle
$$


TABLE 4 continued

3. $O=A\left(i i^{\prime}\right)_{I_{p}}^{\left(\tilde{\omega}_{p}\right)},\left(\tilde{\omega}_{p}\right)=(02),(10) ; \quad u^{\prime}=u-2$

$\left.M=(-1)^{\hat{\sigma}-\hat{\sigma}^{\prime}+\hat{\omega}^{-} \hat{\omega}^{\prime}} \delta_{[n]\left[n^{\prime}\right]} U\left(\left[\tilde{\omega}_{p}\right][\sigma]\left[\omega^{\prime}\right][n] ;\left[\sigma^{\prime}\right] \rho_{-}^{\prime} ;[\omega] \rho_{-}\right)\left\langle\left[\sigma^{\prime}\right] ; I^{\prime} \| A\left(i i^{\prime}\right)\right)_{I_{p}}^{\left(\tilde{\omega}_{p}\right)} \|[\sigma] ; I\right\rangle$

4. $O=P\left(i i^{\prime}\right)_{p}^{(p)}\left(\omega_{0}\right) ; u^{\prime}=u-2 ;\left(\omega_{0}\right)=(00),(11)$ for $(p)=(\mathrm{s}) ;\left(\omega_{0}\right)=(11)$ for $(p)=(\mathrm{a})$;

$$
M=(-1)^{\hat{\omega}_{p}} \boldsymbol{c}_{\left(\omega_{0}\right)}^{(p)}\left[\begin{array}{cccc}
{[\sigma]} & {[n]} & {[\omega]} & \rho \\
{\left[\tilde{\omega}_{p}\right]} & {\left[\omega_{p}\right]} & {\left[\omega_{0}\right]} & - \\
{\left[\sigma^{\prime}\right]} & {\left[n^{\prime}\right]} & {\left[\omega^{\prime}\right]} & \rho^{\prime} \\
- & - & \rho_{0}
\end{array}\right]\left(\left[n^{\prime}\right]\|z\|[n]\right)\left\langle\left[\sigma^{\prime}\right] ; I^{\prime}\left\|A\left(i^{\prime}\right)_{i_{p}}^{\left(\tilde{\omega}_{p}\right)}\right\|[\sigma \sigma] ; I\right\rangle
$$

5. $\left.\boldsymbol{O}=A^{\dagger}\left(i i^{\prime}\right)\right)_{l_{p}}^{\left(\omega_{p}\right)},\left(\omega_{p}\right)=(20),(01) ; \quad u^{\prime}=u-2$

$$
M=\sum_{\left[n_{0}\right]} g_{\left(n_{0}\right)}^{(p)}\left[\begin{array}{cccc}
{[\sigma]} & {[n]} & {[\omega]} & \rho \\
{\left[\tilde{\omega}_{p}\right]} & {\left[n_{0}\right]} & {\left[\omega_{p}\right]} & - \\
{\left[\sigma^{\prime}\right]} & {\left[n^{\prime}\right]} & {\left[\omega^{\prime}\right]} & \rho^{\prime} \\
- & - & - &
\end{array}\right]\left(\left[n^{\prime}\right]\left\|Z^{\left[n_{0}\right]}\right\|[n]\right)\left\langle\left[\sigma^{\prime}\right] ; I^{\prime}\left\|A\left(i i^{\prime}\right)^{\left(\tilde{\omega}_{p^{\prime}}\right)}\right\|[\sigma] ; I\right\rangle
$$

with

$$
\left(\left[n^{\prime}\right]\left\|Z^{\left[n_{0}\right]}\right\|[n]\right)=\frac{1}{\sqrt{2}} \sum_{\left[n^{\prime \prime}\right]}\left(\left[n^{\prime}\right]\|z\|\left[n^{\prime \prime}\right]\right)\left(\left[n^{\prime \prime}\right]\|z\| n\right) U\left([n][2]\left[n^{\prime}\right][2] ;\left[n^{\prime \prime}\right]\left[n_{0}\right]\right)
$$

$$
g_{(40)}^{(s)}=2 \sqrt{5}, g_{(02)}^{(s)}=-\sqrt{2} ; \quad g_{(02)}^{(\mathrm{a})}=2 \sqrt{3}
$$

6. $\boldsymbol{O}=\boldsymbol{A}\left(i^{\prime}\right)_{p_{p}}^{\left(\tilde{\omega}_{p}\right)} ; \quad u^{\prime}=u$

$$
M=\sum_{[\bar{\sigma}] \bar{\rho}} \sqrt{\frac{\operatorname{dim}[\omega]}{\operatorname{dim}\left[\omega^{\prime}\right]} \frac{\operatorname{dim}\left[\sigma^{\prime}\right]}{\operatorname{dim}[\bar{\sigma}]}} U\left(\left[\omega_{p}\right]\left[\sigma^{\prime}\right][\omega]\left[n^{\prime}\right] ;[\bar{\sigma}]_{-} \bar{\rho} ;\left[\omega^{\prime}\right] \rho_{-}^{\prime}\right)
$$

$\left.\times U\left([\sigma][2][\omega]\left[n^{\prime}\right] ;[\bar{\sigma}]_{-} \bar{\rho} ;[n]\right]_{-} \rho\right)\left([n]\|z\|\left[n^{\prime}\right]\right)\left(\left[\sigma^{\prime}\right] ; I^{\prime}\left\|\mathbb{A}\left(i i^{\prime}\right) i_{p}^{\left(\tilde{\omega}_{p}\right)}\right\|[[2] \times[\sigma]][\bar{\sigma}] ; I\right)$

7. $\boldsymbol{O}=\boldsymbol{P}\left(i i^{\prime}\right)_{I_{p}}^{(p)\left(\omega_{0}\right)} ;\left(\omega_{0}\right)=(00),(11)$ for $(p)=(\mathrm{s}) ; \quad(11)$ for $(p)=(\mathrm{a}), u^{\prime}=u$

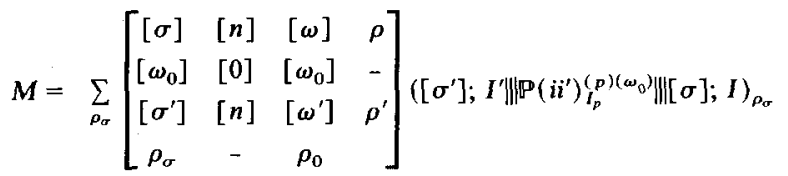

$$
\begin{aligned}
& +c_{\left(\omega_{0}\right)}^{(p)} \sum_{\left[\omega^{\prime \prime}\right]\left[n^{\prime \prime}\right] \rho^{\prime \prime}} U\left([\omega][2]\left[\omega^{\prime}\right]\left[\tilde{\omega}_{p}\right] ;\left[\omega^{\prime \prime}\right]_{--;}\left[\omega_{0}\right]_{-} \rho_{0}\right) \\
& \times U\left([\sigma][n]\left[\omega^{\prime \prime}\right][2] ;[\omega] \rho_{-} ;\left[n^{\prime \prime}\right]-\rho^{\prime \prime}\right)\left(\left[n^{\prime \prime}\right]\|z\|[n]\right) \\
& \times \sum_{[\tilde{\sigma}] \tilde{\rho}} \sqrt{\frac{\operatorname{dim}\left[\omega^{\prime \prime}\right]}{\operatorname{dim}\left[\omega^{\prime}\right]} \frac{\operatorname{dim}\left[\sigma^{\prime}\right]}{\operatorname{dim}[\bar{\sigma}]}} U\left(\left[\omega_{p}\right]\left[\sigma^{\prime}\right]\left[\omega^{\prime \prime}\right]\left[n^{\prime}\right] ;[\bar{\sigma}]_{-} \bar{\rho} ;\left[\omega^{\prime}\right] \rho_{-}^{\prime}\right) \\
& \times U\left([\sigma][2]\left[\omega^{\prime \prime}\right]\left[n^{\prime}\right] ;[\bar{\sigma}]_{-} \bar{\rho} ;\left[n^{\prime \prime}\right]_{-} \rho^{\prime \prime}\right)\left(\left[n^{\prime \prime}\right]\|z\|\left[n^{\prime}\right]\right)\left(\left[\sigma^{\prime}\right] ; I^{\prime} \| \mathbb{A}\left(i i^{\prime}\right)\right\}_{p}^{\left(\tilde{\omega}_{p}\right)} \|[[[2] \times[\sigma]][\bar{\sigma}] ; I)
\end{aligned}
$$$$
\text { where } \quad c_{(00)}^{(\mathrm{s})}=-2, c_{(11)}^{(\mathrm{s})}=-\sqrt{\frac{5}{2}} ; c_{(11)}^{(\mathrm{a})}=+\sqrt{\frac{3}{2}}
$$ 
this investigation. For easy reference, eq. (42) is also included as entry 1 of this table, but now for the most general case for which the product $[\sigma] \times[n] \rightarrow[\omega]$ might involve the multiplicity label, $\rho$. The $k$-space $\mathrm{SU}(3), i$-space $\mathrm{SU}(2)$ reduced matrix elements of $b^{\dagger}, b$ for the case $u^{\prime}=u-1$ can thus be evaluated in terms of SU(3) recoupling coefficients which are readily available through the code of ref. ${ }^{18}$ ) and the $K$-matrix elements of VCS theory. The needed $K$-matrix elements are evaluated by the techniques of ref. ${ }^{14}$ ). For states with $u=0,1,2$ very specific analytic formulae are given in ref. $\left.{ }^{14}\right)$. Note also that for most of the states with $u=0,1,2$ the $[n]$ is uniquely specified by $[\sigma]$ and $[\omega]$, the $K$-matrices are one-dimensional, and no $[n]$-sums are needed in eq. (42).

Matrix elements of $b^{\dagger}$ and $b$ for the case $u^{\prime}=u+1$ could be obtained directly with the use of eqs. (36) and (39). However, the simplest expressions are obtained through the hermitian conjugation of the matrix elements for the case $u^{\prime}=u-1$.

$$
\begin{aligned}
\left\langle\left[\sigma^{\prime}\right]\left[\omega^{\prime}\right] \nu^{\prime} ; I^{\prime}\left\|b_{i}^{\dagger}\right\|[\sigma][\omega] \nu ; I\right\rangle= & \sqrt{\frac{(2 I+1)}{\left(2 I^{\prime}+1\right)}}(-1)^{I+i-I^{\prime}} \sqrt{\frac{\operatorname{dim}[\omega]}{\operatorname{dim}\left[\omega^{\prime}\right]}}(-1)^{\hat{\omega}+1-\hat{\omega}^{\prime}} \\
& \times\left\langle[\sigma][\omega] \nu ; I\left\|\tilde{b}_{i}\right\|\left[\sigma^{\prime}\right]\left[\omega^{\prime}\right] \nu^{\prime} ; I^{\prime}\right\rangle,
\end{aligned}
$$

where the $\mathrm{U}(3)$ or equivalent $\mathrm{SU}(3)$ dimension factor is given by $\operatorname{dim}[\omega]=$ $\frac{1}{2}\left(\lambda_{\omega}+1\right)\left(\mu_{\omega}+1\right)\left(\lambda_{\omega}+\mu_{\omega}+2\right)$. The phase factors follow from the $1 \rightarrow 3$ interchange symmetry property of the $k$-space SU(3) Wigner coefficients and the $i$-space angular momentum Wigner coefficients. In the case of SU(3) these have the simple value shown for the multiplicity-free coupling $(\omega) \times(10) \rightarrow\left(\omega^{\prime}\right)$. [For the definition of the phase $\hat{\omega}$, see eq. (10).]

Since the operators $b_{i}^{\dagger}, b_{i}$ span the six-dimensional $\mathrm{Sp}(6)$ representation (100), the reduced matrix elements of these single-nucleon operators can be expressed in their most convenient form through a set of $\mathrm{Sp}(6) \supset \mathrm{U}(3)$ reduced Wigner coefficients for the $\operatorname{Sp}(6)$ coupling $\left(\Omega_{1} \Omega_{2} \Omega_{3}\right) \times(100) \rightarrow\left(\Omega_{1}^{\prime} \Omega_{2}^{\prime} \Omega_{3}^{\prime}\right)$ [see eq. (5)]. For the case $u^{\prime}=u-1$ the reduced matrix elements of $b_{i}^{\dagger}$ and $b_{i}$ (entries 1 and 2 of table 4) can be expressed as a product of a $S p(6) \supset U(3)$ reduced Wigner coefficient and a $S p(6)$ reduced matrix element. There is, however, no need to introduce a special new notation (such as a quadruple-barred matrix element!) for this $S p(6)$ fully reduced matrix element. For the case $u^{\prime}=u-1$ it is simply the starting matrix element, $\left\langle\left[\sigma^{\prime}\right] ; I^{\prime}\left\|\tilde{b_{i}}\right\|[\sigma] ; I\right\rangle$; i.e.

$$
\begin{aligned}
&\left\langle\left[\sigma^{\prime}\left[\omega^{\prime}\right] \nu^{\prime} ; I^{\prime}\left\|\tilde{b_{i}}\right\|[\sigma][\omega] \nu ; I\right\rangle\right. \\
&=\left\langle\left(\Omega_{1} \Omega_{2} \Omega_{3}\right)[\sigma][\omega] \nu ;(100)[00-1] \|\left(\Omega_{1}^{\prime} \Omega_{2}^{\prime} \Omega_{3}^{\prime}\right)\left[\sigma^{\prime}\right]\left[\omega^{\prime}\right] \nu^{\prime}\right\rangle \\
& \times\left\langle\left[\sigma^{\prime}\right] ; I^{\prime}\left\|\tilde{b_{i}}\right\|[\sigma] ; I\right\rangle .
\end{aligned}
$$

Here, the double-barred coefficient is the $\mathrm{Sp}(6) \supset \mathrm{U}(3)$ reduced Wigner coefficient. This follows from the fact that this coefficient describes a unique $1 \times 1$ unitary transformation for the special case $\left[\omega^{\prime}\right]=\left[\sigma^{\prime}\right]\left(\nu^{\prime}=[0]\right),[\omega]=[\sigma](\nu=[0]), u^{\prime}=$ 
$u-1$; and is chosen to have the value +1 . Note also that both entries 1 and 2 of table 4 are proportional to the $\operatorname{Sp}(6)$ reduced matrix element $\left\langle\left[\sigma^{\prime}\right] ; I^{\prime}\left\|\tilde{b_{i}}\right\|[\sigma] ; I\right\rangle$. It is therefore easy to read off the orthonormal $\mathrm{Sp}(6) \supset \mathrm{U}(3)$ reduced Wigner coefficients for the case $u^{\prime}=u-1$; or, vice versa, from the reduced Wigner coefficients to obtain the matrix elements of $\tilde{b}_{i}$ and $b_{i}^{\dagger}$ by using eq. (44a) or its companion

$$
\begin{aligned}
& u^{\prime}=u-1: \quad\left\langle\left[\sigma^{\prime}\right]\left[\omega^{\prime}\right] \nu^{\prime} ; I^{\prime}\left\|b_{i}^{\dagger}\right\|[\sigma][\omega] \nu ; I\right\rangle \\
&=\left\langle\left(\Omega_{1} \Omega_{2} \Omega_{3}\right)[\sigma][\omega] \nu ;(100)[100] \|\left(\Omega_{1}^{\prime} \Omega_{2}^{\prime} \Omega_{3}^{\prime}\right)\left[\sigma^{\prime}\right]\left[\omega^{\prime}\right] \nu^{\prime}\right\rangle \\
& \times\left\langle\left[\sigma^{\prime}\right] ; I^{\prime}\left\|\tilde{b}_{i}\right\|[\sigma] ; I\right\rangle .
\end{aligned}
$$

The conjugation relation of eq. (43) can be translated into the symmetry relation for the $\mathrm{Sp}(6) \supset \mathrm{U}(3)$ reduced Wigner coefficient

$$
\begin{aligned}
&\left\langle\left(\Omega_{1} \Omega_{2} \Omega_{3}\right)[\sigma][\omega] \nu ;(100)\left[\sigma^{\prime \prime}\right] \|\left(\Omega_{1}^{\prime} \Omega_{2}^{\prime} \Omega_{3}^{\prime}\right)\left[\sigma^{\prime}\right]\left[\omega^{\prime}\right] \nu^{\prime}\right\rangle \\
&= \pm(-1)^{\hat{\omega}-\hat{\omega}^{\prime}} \sqrt{\frac{\operatorname{dim}\left(\Omega_{1}^{\prime} \Omega_{2}^{\prime} \Omega_{3}^{\prime}\right) \operatorname{dim}[\omega]}{\operatorname{dim}\left(\Omega_{1} \Omega_{2} \Omega_{3}\right) \operatorname{dim}\left[\omega^{\prime}\right]}} \\
& \times\left\langle\left(\Omega_{1}^{\prime} \Omega_{2}^{\prime} \Omega_{3}^{\prime}\right)\left[\sigma^{\prime}\right]\left[\omega^{\prime}\right] \nu^{\prime} ;(100)\left[\tilde{\sigma}^{\prime \prime}\right] \|\left(\Omega_{1} \Omega_{2} \Omega_{3}\right)[\sigma][\omega] \nu\right\rangle,
\end{aligned}
$$

with upper (lower) sign for $\left[\sigma^{\prime \prime}\right]=[100]([00-1])$.

The symmetry relation (45) is somewhat dependent on phase choices for the $S p(6)$ states. Eq. (43), on the other hand, is completely independent of such choices and depends solely on the standard angular momentum phase conventions and the SU(3) phase conventions of the computer code of ref. ${ }^{18}$ ). For the case $\boldsymbol{u}^{\prime}=\boldsymbol{u}+\mathbf{1}$ the $\mathrm{Sp}(6)$ fully reduced matrix element has the more complicated value

$$
\sqrt{\frac{\operatorname{dim}\left(\Omega_{1} \Omega_{2} \Omega_{3}\right) \operatorname{dim}\left[\sigma^{\prime}\right]}{\operatorname{dim}\left(\Omega_{1}^{\prime} \Omega_{2}^{\prime} \Omega_{3}^{\prime}\right) \operatorname{dim}[\sigma]}}(-1)^{\hat{\sigma}-\hat{\sigma}^{\prime}}\left\langle\left[\sigma^{\prime}\right] ; I^{\prime}\left\|b_{i}^{\dagger}\right\|[\sigma] ; I\right\rangle .
$$

The $\mathrm{Sp}(6) \supset \mathrm{U}(3)$ reduced Wigner coefficients for the couplings $\left(\Omega_{1} \Omega_{2} \Omega_{3}\right) \times(100) \rightarrow$ $\left(\Omega_{1}^{\prime} \Omega_{2}^{\prime} \Omega_{3}^{\prime}\right.$ ) for states with heritage $u$ and $u^{\prime}=0,1,2$ can be given in very specific analytic form. These are collected in tables 5-11. In these tables, as well as in eqs. (44), (45) the $U(3)$ representations for the single-nucleon creation and annihilation operators are given in their full $\left[\sigma_{1}^{\prime \prime} \sigma_{2}^{\prime \prime} \sigma_{3}^{\prime \prime}\right]$ form; $[100]$ for $b_{i}^{\dagger},[00-1]$ for $b_{i}$. This has the advantage that $\sum_{i} \omega_{i}^{\prime}=\sum_{i}\left(\omega_{i}+\sigma_{i}^{\prime \prime}\right)$ in tables 5-11.

\section{Matrix elements of one-body and pair operators}

One-body operators, $P\left(i i^{\prime}\right)$, and nucleon pair creation and annihilation operators, $A^{\dagger}\left(i i^{\prime}\right), A\left(i i^{\prime}\right)$, can connect states of heritage $u=\sigma_{1}+\sigma_{2}+\sigma_{3}$ to states with $u^{\prime}=$ $u \pm 2, u$. VCS theory again leads to the simplest formulae for states with $u^{\prime}=u-2$. Detailed formulae are therefore given only for states with $u^{\prime}=u-2$ and $u^{\prime}=u$. The remaining cases can again be obtained from hermitian conjugation. Although SU(3) outer multiplicities will not be encountered in most formulae involving heritage 
TABLE 5

$\left\langle\left(\frac{\Omega}{3} \frac{\Omega}{3} \frac{\Omega}{3}-1\right)[1][\omega] ;(100)\left[\sigma^{\prime \prime}\right] \|\left(\frac{\Omega}{3} \frac{\Omega}{3} \frac{\Omega}{3}\right)[0][n]\right\rangle$

\begin{tabular}{ccc}
\hline$[\omega]$ & {$\left[\sigma^{\prime \prime}\right]$} & $\langle\|\rangle$ \\
{$\left[n_{1}-1 n_{2} n_{3}\right]$} & {$[100]$} & $-\sqrt{\frac{\left(n_{1}+2\right)\left(n_{1}-n_{2}\right)\left(n_{1}-n_{3}+1\right)}{2 \Omega\left(n_{1}-n_{2}+1\right)\left(n_{1}-n_{3}+2\right)}}$ \\
{$\left[n_{1} n_{2}-1 n_{3}\right]$} & {$[100]$} & $\sqrt{\frac{\left(n_{2}+1\right)\left(n_{1}-n_{2}+2\right)\left(n_{2}-n_{3}\right)}{2 \Omega\left(n_{1}-n_{2}+1\right)\left(n_{2}-n_{3}+1\right)}}$ \\
{$\left[n_{1} n_{2} n_{3}-1\right]$} & {$[100]$} & $-\sqrt{\frac{n_{3}\left(n_{1}-n_{3}+3\right)\left(n_{2}-n_{3}+2\right)}{2 \Omega\left(n_{1}-n_{3}+2\right)\left(n_{2}-n_{3}+1\right)}}$ \\
{$\left[n_{1}+1 n_{2} n_{3}\right]$} & {$[00-1]$} & $\sqrt{\frac{\left(\frac{2}{3} \Omega-n_{1}\right)\left(n_{1}-n_{2}+2\right)\left(n_{1}-n_{3}+3\right)}{2 \Omega\left(n_{1}-n_{2}+1\right)\left(n_{1}-n_{3}+2\right)}}$ \\
{$\left[n_{2} n_{2}+1 n_{3}\right]$} & {$[00-1]$} & $\sqrt{\frac{\left(\frac{2}{3} \Omega-n_{2}+1\right)\left(n_{1}-n_{2}\right)\left(n_{2}-n_{3}+2\right)}{2 \Omega\left(n_{1}-n_{2}+1\right)\left(n_{2}-n_{3}+1\right)}}$ \\
{$\left[n_{1} n_{2} n_{3}+1\right]$} & {$[00-1]$} & $\sqrt{\frac{\left(\frac{2}{3} \Omega-n_{3}+2\right)\left(n_{1}-n_{3}+1\right)\left(n_{2}-n_{3}\right)}{2 \Omega\left(n_{1}-n_{3}+2\right)\left(n_{2}-n_{3}+1\right)}}$ \\
\hline
\end{tabular}

TABle 6

$\left\langle\left(\frac{\Omega}{3} \frac{\Omega}{3} \frac{\Omega}{3}\right)[0][\omega] ;(100)\left[\sigma^{\prime \prime}\right] \|\left(\frac{\Omega}{3} \frac{\Omega}{3} \frac{\Omega}{3}-1\right)[1]\left[\omega^{\prime}\right] \nu^{\prime} \equiv\left[n^{\prime}\right]\right\rangle$

(1) $\left[\omega^{\prime}\right]=\left[n_{1}^{\prime}+1 n_{2}^{\prime} n_{3}^{\prime}\right]$

$$
\begin{aligned}
& {[\omega]=[n]=\left[\begin{array}{lll}
n_{1}^{\prime} & n_{2}^{\prime} & n_{3}^{\prime}
\end{array}\right] ;\left[\begin{array}{ll}
\sigma^{\prime \prime}
\end{array}\right]=\left[\begin{array}{lll}
1 & 0 & 0
\end{array}\right]} \\
& +\sqrt{\frac{\left(2 \Omega-3 n_{1}^{\prime}\right)}{2(\Omega+6)}} \\
& {[\omega]=[n]=\left[n_{1}^{\prime}+2 n_{2}^{\prime} n_{3}^{\prime}\right] ;\left[\sigma^{\prime \prime}\right]=[00-1]} \\
& +\sqrt{\frac{3\left(n_{1}^{\prime}+4\right)}{2(\Omega+6)}}
\end{aligned}
$$

(2) $\left[\omega^{\prime}\right]=\left[\begin{array}{lll}n_{1}^{\prime} & n_{2}^{\prime}+1 & n_{3}^{\prime}\end{array}\right]$

$$
\begin{aligned}
& {[\omega]=[n]=\left[\begin{array}{lll}
n_{1}^{\prime} & n_{2}^{\prime} & n_{3}^{\prime}
\end{array}\right] ;\left[\sigma^{\prime \prime}\right]=\left[\begin{array}{lll}
1 & 0 & 0
\end{array}\right]} \\
& {[\omega]=[n]=\left[\begin{array}{lll}
n_{1}^{\prime} & n_{2}^{\prime}+2 & n_{3}^{\prime}
\end{array}\right] ;\left[\sigma^{\prime \prime}\right]=\left[\begin{array}{ll}
0 & 0-1
\end{array}\right]}
\end{aligned}
$$$$
-\sqrt{\frac{\left(2 \Omega-3 n_{2}^{\prime}+3\right)}{2(\Omega+6)}}
$$$$
+\sqrt{\frac{3\left(n_{2}^{\prime}+3\right)}{2(\Omega+6)}}
$$

(3) $\left[\omega^{\prime}\right]=\left[n_{1}^{\prime} n_{2}^{\prime} n_{3}^{\prime}+1\right]$

$$
\begin{aligned}
& {[\omega]=[n]=\left[\begin{array}{lll}
n_{1}^{\prime} & n_{2}^{\prime} & n_{3}^{\prime}
\end{array}\right] ;\left[\sigma^{\prime \prime}\right]=\left[\begin{array}{lll}
1 & 0 & 0
\end{array}\right]} \\
& {[\omega]=[n]=\left[\begin{array}{lll}
n_{1}^{\prime} & n_{2}^{\prime} & n_{3}^{\prime}+2
\end{array}\right] ;\left[\sigma^{\prime \prime}\right]=\left[\begin{array}{lll}
0 & 0-1
\end{array}\right]}
\end{aligned}
$$$$
+\sqrt{\frac{\left(2 \Omega-3 n_{3}^{\prime}+6\right)}{2(\Omega+6)}}
$$$$
+\sqrt{\frac{3\left(n_{3}^{\prime}+2\right)}{2(\Omega+6)}}
$$ 
TABLE 7

$\left\langle\left(\frac{\Omega}{3} \frac{\Omega}{3} \frac{\Omega}{3}-1\right)[1][\omega] \nu \equiv[n] ;(100)\left[\sigma^{\prime \prime}\right] \|\left(\frac{\Omega}{3} \frac{\Omega}{3}-1 \frac{\Omega}{3}-1\right)[11]\left[\omega^{\prime}\right] \nu^{\prime} \equiv\left[n^{\prime}\right]\right\rangle$

$[\omega]$

[n]

$\left[\sigma^{\prime \prime}\right]$

$\langle\|\rangle$

(1) $\left[\omega^{\prime}\right]=\left[n_{1}^{\prime}+1 n_{2}^{\prime}+1 n_{3}^{\prime}\right]$

$\begin{array}{cccc}{\left[n_{1}^{\prime}+1 n_{2}^{\prime}+2 n_{3}^{\prime}\right]} & {\left[n_{1}^{\prime} n_{2}^{\prime}+2 n_{3}^{\prime}\right]} & {[00-1]} & \sqrt{\frac{\left(n_{2}^{\prime}+3\right)\left(n_{1}^{\prime}-n_{2}^{\prime}\right)}{2\left(\frac{2}{3} \Omega+5\right)\left(n_{1}^{\prime}-n_{2}^{\prime}+1\right)}} \\ {\left[n_{1}^{\prime}+2 n_{2}^{\prime}+1 n_{3}^{\prime}\right]} & {\left[n_{1}^{\prime}+2 n_{2}^{\prime} n_{3}^{\prime}\right]} & {[00-1]} & -\sqrt{\frac{\left(n_{1}^{\prime}+4\right)\left(n_{1}^{\prime}-n_{2}^{\prime}+2\right)}{2\left(\frac{2}{3} \Omega+5\right)\left(n_{1}^{\prime}-n_{2}^{\prime}+1\right)}} \\ {\left[n_{1}^{\prime}+1 n_{2}^{\prime} n_{3}^{\prime}\right]} & {\left[n_{1}^{\prime} n_{2}^{\prime} n_{3}^{\prime}\right]} & {\left[\begin{array}{lll}1 & 0 & 0\end{array}\right.} & -\sqrt{\frac{\left(\frac{2}{3} \Omega-n_{2}^{\prime}+1\right)\left(n_{1}^{\prime}-n_{2}^{\prime}+2\right)}{2\left(\frac{2}{3} \Omega+5\right)\left(n_{1}^{\prime}-n_{2}^{\prime}+1\right)}} \\ {\left[n_{1}^{\prime} n_{2}^{\prime}+1 n_{3}^{\prime}\right]} & {\left[n_{1}^{\prime} n_{2}^{\prime} n_{3}^{\prime}\right]} & {[100]} & -\sqrt{\frac{\left(\frac{2}{3} \Omega-n_{1}^{\prime}\right)\left(n_{1}^{\prime}-n_{2}^{\prime}\right)}{2\left(\frac{2}{3} \Omega+5\right)\left(n_{1}^{\prime}-n_{2}^{\prime}+1\right)}}\end{array}$

(2) $\left[\omega^{\prime}\right]=\left[n_{1}^{\prime}+1 n_{2}^{\prime} n_{3}^{\prime}+1\right]$

$\begin{array}{cccc}{\left[n_{1}^{\prime}+2 n_{2}^{\prime} n_{3}^{\prime}+1\right]} & {\left[n_{1}^{\prime}+2 n_{2}^{\prime} n_{3}^{\prime}\right]} & {[00-1]} & -\sqrt{\frac{\left(n_{1}^{\prime}+4\right)\left(n_{1}^{\prime}-n_{3}^{\prime}+3\right)}{2\left(\frac{2}{3} \Omega+5\right)\left(n_{1}^{\prime}-n_{3}^{\prime}+2\right)}} \\ {\left[n_{1}^{\prime}+1 n_{2}^{\prime} n_{3}^{\prime}+2\right]} & {\left[n_{1}^{\prime} n_{2}^{\prime} n_{3}^{\prime}+2\right]} & {[00-1]} & \sqrt{\frac{\left(n_{3}^{\prime}+2\right)\left(n_{1}^{\prime}-n_{3}^{\prime}+1\right)}{2\left(\frac{2}{3} \Omega+5\right)\left(n_{1}^{\prime}-n_{3}^{\prime}+2\right)}} \\ {\left[n_{1}^{\prime}+1 n_{2}^{\prime} n_{3}^{\prime}\right]} & {\left[n_{1}^{\prime} n_{2}^{\prime} n_{3}^{\prime}\right]} & {\left[\begin{array}{lll}1 & 0 & 0\end{array}\right.} & \sqrt{\frac{\left(\frac{2}{3} \Omega-n_{3}^{\prime}+2\right)\left(n_{1}^{\prime}-n_{3}^{\prime}+3\right)}{2\left(\frac{2}{3} \Omega+5\right)\left(n_{1}^{\prime}-n_{3}^{\prime}+2\right)}} \\ {\left[n_{1}^{\prime} n_{2}^{\prime} n_{3}^{\prime}+1\right]} & {\left[n_{1}^{\prime} n_{2}^{\prime} n_{3}^{\prime}\right]} & {\left[\begin{array}{lll}1 & 0 & 0\end{array}\right.} & -\sqrt{\frac{\left(\frac{2}{3} \Omega-n_{1}^{\prime}\right)\left(n_{1}^{\prime}-n_{3}^{\prime}+1\right)}{2\left(\frac{2}{3} \Omega+5\right)\left(n_{1}^{\prime}-n_{3}^{\prime}+2\right)}}\end{array}$

(3) $\left[\omega^{\prime}\right]=\left[n_{1}^{\prime} n_{2}^{\prime}+1 n_{3}^{\prime}+1\right]$

$\begin{array}{cccc}{\left[n_{1}^{\prime} n_{2}^{\prime}+2 n_{3}^{\prime}+1\right]} & {\left[n_{1}^{\prime} n_{2}^{\prime}+2 n_{3}^{\prime}\right]} & {[00-1]} & -\sqrt{\frac{\left(n_{2}^{\prime}+3\right)\left(n_{2}^{\prime}-n_{3}^{\prime}+2\right)}{2\left(\frac{2}{3} \Omega+5\right)\left(n_{2}^{\prime}-n_{3}^{\prime}+1\right)}} \\ {\left[n_{1}^{\prime} n_{2}^{\prime}+1 n_{3}^{\prime}+2\right]} & {\left[n_{1}^{\prime} n_{2}^{\prime} n_{3}^{\prime}+2\right]} & {[00-1]} & \sqrt{\frac{\left(n_{3}^{\prime}+2\right)\left(n_{2}^{\prime}-n_{3}^{\prime}\right)}{2\left(\frac{2}{3} \Omega+5\right)\left(n_{2}^{\prime}-n_{3}^{\prime}+1\right)}} \\ {\left[n_{1}^{\prime} n_{2}^{\prime}+1 n_{3}^{\prime}\right]} & {\left[n_{1}^{\prime} n_{2}^{\prime} n_{3}^{\prime}\right]} & {\left[\begin{array}{lll}1 & 0\end{array}\right]} & \sqrt{\frac{\left(\frac{2}{3} \Omega-n_{3}^{\prime}+2\right)\left(n_{2}^{\prime}-n_{3}^{\prime}+2\right)}{2\left(\frac{2}{3} \Omega+5\right)\left(n_{2}^{\prime}-n_{3}^{\prime}+1\right)}} \\ {\left[n_{1}^{\prime} n_{2}^{\prime} n_{3}^{\prime}+1\right]} & {\left[n_{1}^{\prime} n_{2}^{\prime} n_{3}^{\prime}\right]} & {\left[\begin{array}{lll}1 & 0\end{array}\right]} & \sqrt{\frac{\left(\frac{2}{3} \Omega-n_{2}^{\prime}+1\right)\left(n_{2}^{\prime}-n_{3}^{\prime}\right)}{2\left(\frac{2}{3} \Omega+5\right)\left(n_{2}^{\prime}-n_{3}^{\prime}+1\right)}}\end{array}$

$u \leqslant 2$, one-body operators $\left[b^{\dagger} \times b\right]$ coupled to the 8-dimensional SU(3) representa. tion $\left(\omega_{0}\right)=(11)$ will lead to multiplicity in their coupling to arbitrary $(\omega)$. The reduced matrix elements of such operators will therefore require the SU(3) multi. plicity label $\rho$. All formulae will therefore be given for the most general intrinsic state $[\sigma]$ for which the coupling $[\sigma] \times[n] \rightarrow[\omega]$ will in general also require the 
TABLE 8

$\left\langle\left(\frac{\Omega}{3} \frac{\Omega}{3}-1 \frac{\Omega}{3}-1\right)[11][\omega] \nu \equiv[n] ;\left(\begin{array}{lll}1 & 0 & 0\end{array}\right)\left[\sigma^{\prime \prime}\right] \|\left(\frac{\Omega}{3} \frac{\Omega}{3} \frac{\Omega}{3}-1\right)[1]\left[\omega^{\prime}\right] \nu^{\prime} \equiv\left[n^{\prime}\right]\right\rangle$

\begin{tabular}{llll}
\hline$[\omega]$ & {$\left[\sigma^{\prime \prime}\right]$} & {$[n]$} & $\|$
\end{tabular}

(1) $\left[\omega^{\prime}\right]=\left[n_{1}^{\prime}+1 \quad n_{2}^{\prime} n_{3}^{\prime}\right]$

$\begin{array}{lccc}{\left[n_{1}^{\prime}+1 n_{2}^{\prime}-1 n_{3}^{\prime}\right]} & {[100]} & {\left[n_{1}^{\prime} n_{2}^{\prime}-2 n_{3}^{\prime}\right]} & \sqrt{\frac{\left(n_{2}^{\prime}+1\right)\left(n_{2}^{\prime}-n_{3}^{\prime}\right)}{2\left(\frac{2}{3} \Omega+1\right)\left(n_{2}^{\prime}-n_{3}^{\prime}+1\right)}} \\ {\left[n_{1}^{\prime}+1 n_{2}^{\prime} n_{3}^{\prime}-1\right]} & {[100]} & {\left[n_{1}^{\prime} n_{2}^{\prime} n_{3}^{\prime}-2\right]} & -\sqrt{\frac{n_{3}^{\prime}\left(n_{2}^{\prime}-n_{3}^{\prime}+2\right)}{2\left(\frac{2}{3} \Omega+1\right)\left(n_{2}^{\prime}-n_{3}^{\prime}+1\right)}} \\ {\left[n_{1}^{\prime}+1 n_{2}^{\prime}+1 n_{3}^{\prime}\right]} & {[00-1]} & {\left[n_{1}^{\prime} n_{2}^{\prime} n_{3}^{\prime}\right]} & \sqrt{\frac{\left(\frac{2}{3} \Omega-n_{2}^{\prime}+1\right)\left(n_{2}^{\prime}-n_{3}^{\prime}+2\right)}{2\left(\frac{2}{3} \Omega+1\right)\left(n_{2}^{\prime}-n_{3}^{\prime}+1\right)}} \\ {\left[n_{1}^{\prime}+1 n_{2}^{\prime} n_{3}^{\prime}+1\right]} & {[00-1]} & {\left[n_{1}^{\prime} n_{2}^{\prime} n_{3}^{\prime}\right]} & \sqrt{\frac{\left(\frac{2}{3} \Omega-n_{3}^{\prime}+2\right)\left(n_{2}^{\prime}-n_{3}^{\prime}\right)}{2\left(\frac{2}{3} \Omega+1\right)\left(n_{2}^{\prime}-n_{3}^{\prime}+1\right)}}\end{array}$

(2) $\left[\omega^{\prime}\right]=\left[n_{1}^{\prime} n_{2}^{\prime}+1 n_{3}^{\prime}\right]$

\begin{tabular}{|c|c|c|c|}
\hline$\left[n_{1}^{\prime}-1 n_{2}^{\prime}+1 n_{3}^{\prime}\right]$ & {$\left[\begin{array}{lll}1 & 0 & 0\end{array}\right]$} & {$\left[n_{1}^{\prime}-2 n_{2}^{\prime} n_{3}^{\prime}\right]$} & $\sqrt{\frac{\left(n_{1}^{\prime}+2\right)\left(n_{1}^{\prime}-n_{3}^{\prime}+1\right)}{2\left(\frac{2}{3} \Omega+1\right)\left(n_{1}^{\prime}-n_{3}^{\prime}+2\right)}}$ \\
\hline$\left[n_{1}^{\prime} n_{2}^{\prime}+1 n_{3}^{\prime}-1\right]$ & {$\left[\begin{array}{lll}1 & 0 & 0\end{array}\right]$} & {$\left[\begin{array}{lll}n_{1}^{\prime} & n_{2}^{\prime} & n_{3}^{\prime}-2\end{array}\right]$} & $-\sqrt{\frac{n_{3}^{\prime}\left(n_{1}^{\prime}-n_{3}^{\prime}+3\right)}{2\left(\frac{2}{3} \Omega+1\right)\left(n_{1}^{\prime}-n_{3}^{\prime}+2\right)}}$ \\
\hline$\left[n_{1}^{\prime}+1 n_{2}^{\prime}+1 n_{3}^{\prime}\right]$ & {$\left[\begin{array}{lll}0 & 0 & -1\end{array}\right]$} & {$\left[\begin{array}{lll}n_{1}^{\prime} & n_{2}^{\prime} & n_{3}^{\prime}\end{array}\right]$} & $-\sqrt{\frac{\left(\frac{2}{3} \Omega-n_{1}^{\prime}\right)\left(n_{1}^{\prime}-n_{3}^{\prime}+3\right)}{2\left(\frac{2}{3} \Omega+1\right)\left(n_{1}^{\prime}-n_{3}^{\prime}+2\right)}}$ \\
\hline$\left[n_{1}^{\prime} n_{2}^{\prime}+1 n_{3}^{\prime}+1\right]$ & {$\left[\begin{array}{lll}0 & 0 & -1\end{array}\right]$} & {$\left[\begin{array}{lll}n_{1}^{\prime} & n_{2}^{\prime} & n_{3}^{\prime}\end{array}\right]$} & $\sqrt{\frac{\left(\frac{2}{3} \Omega-n_{3}^{\prime}+2\right)\left(n_{1}^{\prime}-n_{3}^{\prime}+1\right)}{2\left(\frac{2}{3} \Omega+1\right)\left(n_{1}^{\prime}-n_{3}^{\prime}+2\right)}}$ \\
\hline
\end{tabular}

(3) $\left[\omega^{\prime}\right]=\left[n_{1}^{\prime} n_{2}^{\prime} n_{3}^{\prime}+1\right]$

$\begin{array}{llll}{\left[n_{1}^{\prime}-1 n_{2}^{\prime} n_{3}^{\prime}+1\right]} & {[100]} & {\left[n_{1}^{\prime}-2 n_{2}^{\prime} n_{3}^{\prime}\right]} & \sqrt{\frac{\left(n_{1}^{\prime}+2\right)\left(n_{1}^{\prime}-n_{2}^{\prime}\right)}{2\left(\frac{2}{3} \Omega+1\right)\left(n_{1}^{\prime}-n_{2}^{\prime}+1\right)}} \\ {\left[n_{1}^{\prime} n_{2}^{\prime}-1 n_{3}^{\prime}+1\right]} & {[100]} & {\left[n_{1}^{\prime} n_{2}^{\prime}-2 n_{3}^{\prime}\right]} & -\sqrt{\frac{\left(n_{2}^{\prime}+1\right)\left(n_{1}^{\prime}-n_{2}^{\prime}+2\right)}{2\left(\frac{2}{3} \Omega+1\right)\left(n_{1}^{\prime}-n_{2}^{\prime}+1\right)}} \\ {\left[n_{1}^{\prime}+1 n_{2}^{\prime} n_{3}^{\prime}+1\right]} & {[00-1]} & {\left[n_{1}^{\prime} n_{2}^{\prime} n_{3}^{\prime}\right]} & -\sqrt{\frac{\left(\frac{2}{3} \Omega-n_{1}^{\prime}\right)\left(n_{1}^{\prime}-n_{2}^{\prime}+2\right)}{2\left(\frac{2}{3} \Omega+1\right)\left(n_{1}^{\prime}-n_{2}^{\prime}+1\right)}} \\ {\left[n_{1}^{\prime} n_{2}^{\prime}+1 n_{3}^{\prime}+1\right]} & {[00-1]} & {\left[n_{1}^{\prime} n_{2}^{\prime} n_{3}^{\prime}\right]} & -\sqrt{\frac{\left(\frac{2}{3} \Omega-n_{2}^{\prime}+1\right)\left(n_{1}^{\prime}-n_{2}^{\prime}\right)}{2\left(\frac{2}{3} \Omega+1\right)\left(n_{1}^{\prime}-n_{2}^{\prime}+1\right)}}\end{array}$

SU(3) multiplicity label $\rho$. Wherever multiplicity labels are not needed in a recoupling coefficient they are to be replaced by a dash. Note also that row and column indices of the $\left(K K^{\dagger}\right)$ matrix for a specific $[\sigma],[\omega]$ are now labeled by both $[n]$ and $\rho$, although $\rho$ will come into play only for certain $[\sigma],[n]$ combinations.

The matrix elements of operators $A\left(i i^{\prime}\right), A^{\dagger}\left(i i^{\prime}\right)$, and $P\left(i i^{\prime}\right)$ for states with $u^{\prime}=u-2$ all three lead to intrinsic reduced matrix elements of the intrinsic operator of type 
TABle 9a

$$
\left\langle\left(\frac{\Omega}{3} \frac{\Omega}{3} \frac{\Omega}{3}-1\right)[1][\omega] \nu \equiv[n] ;(100)\left[\sigma^{\prime \prime}\right] \|\left(\frac{\Omega}{3} \frac{\Omega}{3} \frac{\Omega}{3}-2\right)[2]\left[\omega^{\prime}\right] \nu^{\prime}\right\rangle
$$

\begin{tabular}{llll}
\hline$\left[\sigma^{\prime \prime}\right]$ & {$[\omega]$} & {$[n]$} & $\langle\|\rangle$ \\
\hline
\end{tabular}

(1) $\left[\omega^{\prime}\right]-\left[n_{1}^{\prime}+1 n_{2}^{\prime}+1 n_{3}^{\prime}\right]\left(\nu^{\prime} \equiv\left[n_{1}^{\prime} n_{2}^{\prime} n_{3}^{\prime}\right]\right)$

$\begin{array}{lccc}{\left[\begin{array}{lll}1 & 0 & 0\end{array}\right]} & {\left[n_{1}^{\prime}+1 n_{2}^{\prime} n_{3}^{\prime}\right]} & {\left[n_{1}^{\prime} n_{2}^{\prime} n_{3}^{\prime}\right]} & -\sqrt{\frac{\left(\frac{2}{3} \Omega-n_{2}^{\prime}+1\right)\left(n_{1}^{\prime}-n_{2}^{\prime}\right)}{\left.\frac{2}{3} \Omega+3\right)\left(n_{1}^{\prime}-n_{2}^{\prime}+1\right)}} \\ {\left[\begin{array}{lll}1 & 0 & 0\end{array}\right]} & {\left[n_{1}^{\prime} n_{2}^{\prime}+1 n_{3}^{\prime}\right]} & {\left[n_{1}^{\prime} n_{2}^{\prime} n_{3}^{\prime}\right]} & \sqrt{\frac{\left(\frac{2}{3} \Omega-n_{1}^{\prime}\right)\left(n_{1}^{\prime}-n_{2}^{\prime}+2\right)}{2\left(\frac{2}{3} \Omega+3\right)\left(n_{1}^{\prime}-n_{2}^{\prime}+1\right)}} \\ {\left[\begin{array}{llll}0 & 0-1\end{array}\right]} & {\left[n_{1}^{\prime}+2 n_{2}^{\prime}+1 n_{3}^{\prime}\right]} & {\left[n_{1}^{\prime}+2 n_{2}^{\prime} n_{3}^{\prime}\right]} & \sqrt{\frac{\left(n_{1}^{\prime}+4\right)\left(n_{1}^{\prime}-n_{2}^{\prime}\right)}{2\left(\frac{2}{3} \Omega+3\right)\left(n_{1}^{\prime}-n_{2}^{\prime}+1\right)}} \\ {\left[\begin{array}{lllll}0 & 0-1\end{array}\right]} & {\left[n_{1}^{\prime}+1 n_{2}^{\prime}+2 n_{3}^{\prime}\right]} & {\left[n_{1}^{\prime} n_{2}^{\prime}+2 n_{3}^{\prime}\right]} & \sqrt{\frac{\left(n_{2}^{\prime}+3\right)\left(n_{1}^{\prime}-n_{2}^{\prime}+2\right)}{2\left(\frac{2}{3} \Omega+3\right)\left(n_{1}^{\prime}-n_{2}^{\prime}+1\right)}}\end{array}$

(2) $\left[\omega^{\prime}\right]=\left[n_{1}^{\prime}+1 n_{2}^{\prime} n_{3}^{\prime}+1\right]\left(\nu^{\prime} \equiv\left[n_{1}^{\prime} n_{2}^{\prime} n_{3}^{\prime}\right]\right)$

\begin{tabular}{|c|c|c|c|}
\hline$\left[\begin{array}{lll}1 & 0 & 0\end{array}\right]$ & {$\left[\begin{array}{lll}n_{1}^{\prime}+1 & n_{2}^{\prime} & n_{3}^{\prime}\end{array}\right]$} & {$\left[\begin{array}{lll}n_{1}^{\prime} & n_{2}^{\prime} & n_{3}^{\prime}\end{array}\right]$} & $\sqrt{\frac{\left(\frac{2}{3} \Omega-n_{3}^{\prime}+2\right)\left(n_{1}^{\prime}-n_{3}^{\prime}+1\right)}{2\left(\frac{2}{3} \Omega+3\right)\left(n_{1}^{\prime}-n_{3}^{\prime}+2\right)}}$ \\
\hline$\left[\begin{array}{lll}1 & 0 & 0\end{array}\right]$ & {$\left[n_{1}^{\prime} n_{2}^{\prime} n_{3}^{\prime}+1\right]$} & {$\left[\begin{array}{lll}n_{1}^{\prime} & n_{2}^{\prime} & n_{3}^{\prime}\end{array}\right]$} & $\sqrt{\frac{\left(\frac{2}{3} \Omega-n_{1}^{\prime}\right)\left(n_{1}^{\prime}-n_{3}^{\prime}+3\right)}{2\left(\frac{2}{3} \Omega+3\right)\left(n_{1}^{\prime}-n_{3}^{\prime}+2\right)}}$ \\
\hline$\left[\begin{array}{lll}0 & 0 & -1\end{array}\right]$ & {$\left[n_{1}^{\prime}+2 n_{2}^{\prime} n_{3}^{\prime}+1\right]$} & {$\left[n_{1}^{\prime}+2 n_{2}^{\prime} n_{3}^{\prime}\right]$} & $\sqrt{\frac{\left(n_{1}^{\prime}+4\right)\left(n_{1}^{\prime}-n_{3}^{\prime}+1\right)}{2\left(\frac{2}{3} \Omega+3\right)\left(n_{1}^{\prime}-n_{3}^{\prime}+2\right)}}$ \\
\hline$\left[\begin{array}{lll}0 & 0 & 1\end{array}\right]$ & {$\left[n_{1}^{\prime}+1 n_{2}^{\prime} n_{3}^{\prime}+2\right]$} & {$\left[n_{1}^{\prime} n_{2}^{\prime} n_{3}^{\prime}+2\right]$} & $\sqrt{\frac{\left(n_{3}^{\prime}+2\right)\left(n_{1}^{\prime}-n_{3}^{\prime}+3\right)}{2\left(\frac{2}{3} \Omega+3\right)\left(n_{1}^{\prime}-n_{3}^{\prime}+2\right)}}$ \\
\hline
\end{tabular}

(3) $\left[\omega^{\prime}\right]=\left[n_{1}^{\prime} n_{2}^{\prime}+1 n_{3}^{\prime}+1\right]\left(\nu^{\prime} \equiv\left[n_{1}^{\prime} n_{2}^{\prime} n_{3}^{\prime}\right]\right)$

$\begin{array}{lccc}{\left[\begin{array}{lll}1 & 0 & 0\end{array}\right]} & {\left[n_{1}^{\prime} n_{2}^{\prime}+1 n_{3}^{\prime}\right]} & {\left[n_{1}^{\prime} n_{2}^{\prime} n_{3}^{\prime}\right]} & \sqrt{\frac{\left(\frac{2}{3} \Omega-n_{3}^{\prime}+2\right)\left(n_{2}^{\prime}-n_{3}^{\prime}\right)}{2\left(\frac{2}{3} \Omega+3\right)\left(n_{2}^{\prime}-n_{3}^{\prime}+1\right)}} \\ {\left[\begin{array}{llll}1 & 0 & 0\end{array}\right]} & {\left[n_{1}^{\prime} n_{2}^{\prime} n_{3}^{\prime}+1\right]} & {\left[n_{1}^{\prime} n_{2}^{\prime} n_{3}^{\prime}\right]} & -\sqrt{\frac{\left(\frac{2}{3} \Omega-n_{2}^{\prime}+1\right)\left(n_{2}^{\prime}-n_{3}^{\prime}+2\right)}{2\left(\frac{2}{3} \Omega+3\right)\left(n_{2}^{\prime}-n_{3}^{\prime}+1\right)}} \\ {\left[\begin{array}{llll}0 & 0 & -1\end{array}\right]} & {\left[n_{1}^{\prime} n_{2}^{\prime}+2 n_{3}^{\prime}+1\right]} & {\left[n_{1}^{\prime} n_{2}^{\prime}+2 n_{3}^{\prime}\right]} & \sqrt{\frac{\left(n_{2}^{\prime}+3\right)\left(n_{2}^{\prime}-n_{3}^{\prime}\right)}{2\left(\frac{2}{3} \Omega+3\right)\left(n_{2}^{\prime}-n_{3}^{\prime}+1\right)}} \\ {\left[\begin{array}{lll}0 & 0 & -1\end{array}\right]} & {\left[n_{1}^{\prime} n_{2}^{\prime}+1 n_{3}^{\prime}+2\right]} & {\left[n_{1}^{\prime} n_{2}^{\prime} n_{3}^{\prime}+2\right]} & \sqrt{\frac{\left(n_{3}^{\prime}+2\right)\left(n_{2}^{\prime}-n_{3}^{\prime}+2\right)}{2\left(\frac{2}{3} \Omega+3\right)\left(n_{2}^{\prime}-n_{3}^{\prime}+1\right)}}\end{array}$

$\mathbb{A}\left(i i^{\prime}\right)$. Since the left action of $\mathbb{A}\left(i i^{\prime}\right)$ on an intrinsic state $\left[\sigma^{\prime}\right]$ with $u^{\prime}=u-2$ connects this $\left[\sigma^{\prime}\right]$ to a purely intrinsic state $[\sigma]$, the full reduced matrix element can be reduced to matrix elements of purely intrinsic and pure $z$-space type via straightforward recoupling techniques, as in eq. (42). The resulting expressions are given as entries 3-5 in table 4. 


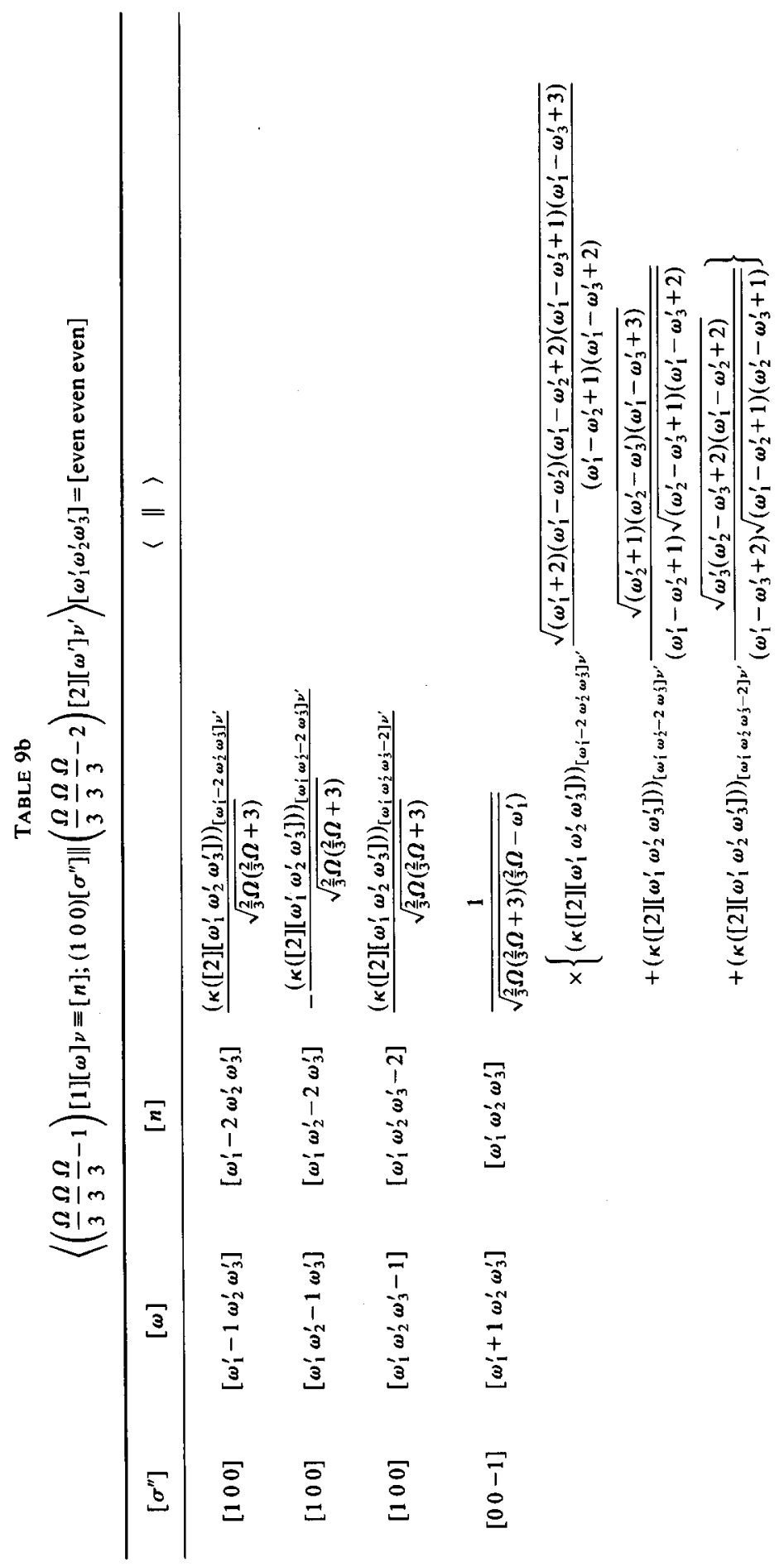



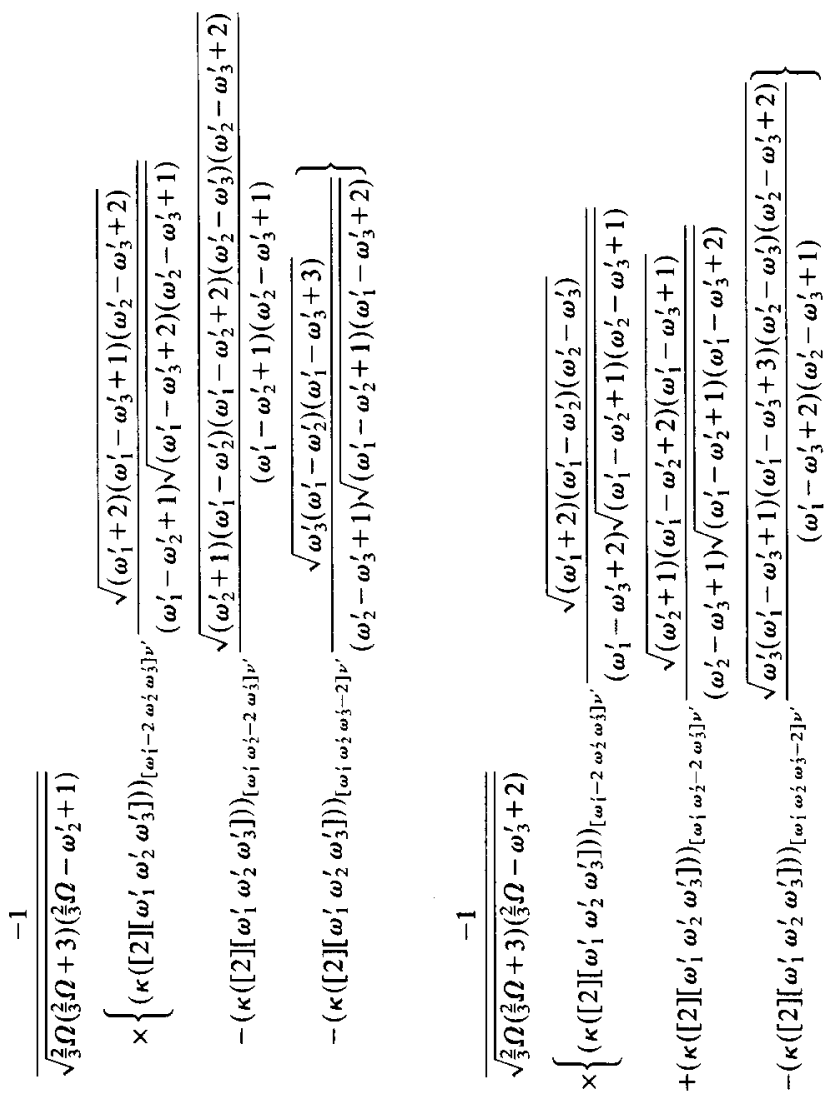

$-m$
3
-13
3
-3
-3
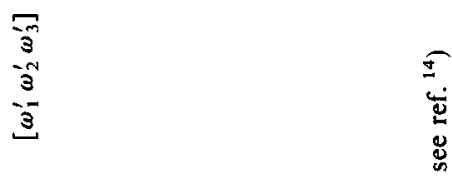

$-m$
3
+
-4
3
-3
3

7
7
-3
-4
-3
-3
-3

$\frac{1=0}{2}$

章

7
0
0

7
0
0 


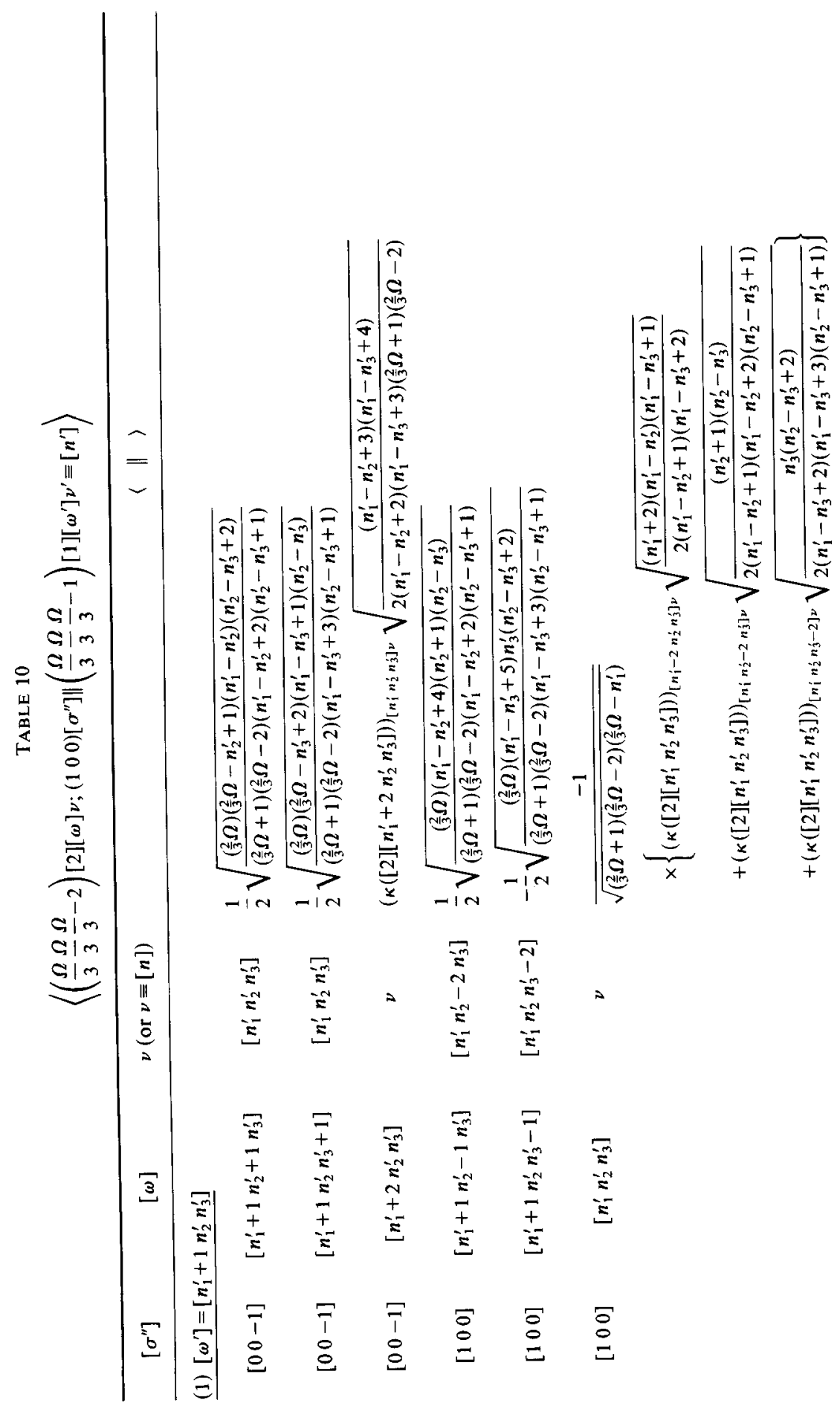

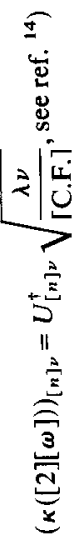



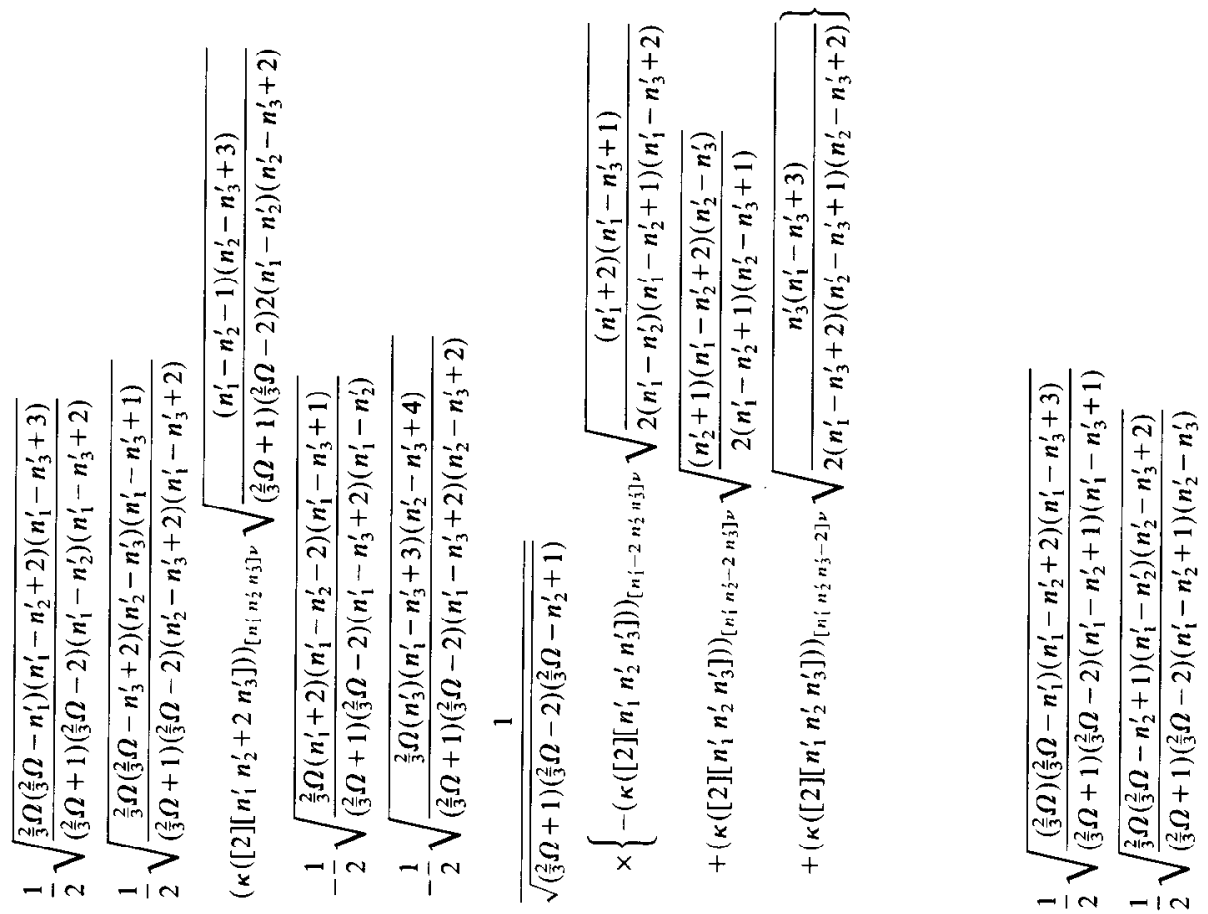

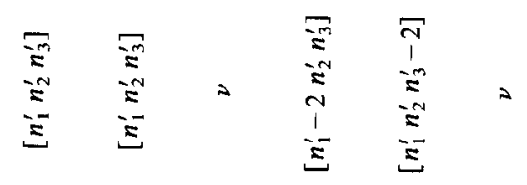

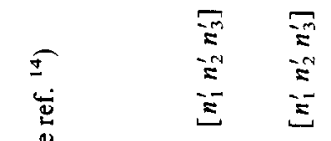

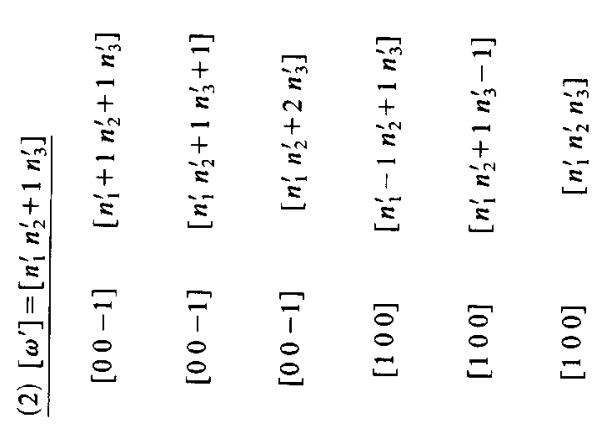

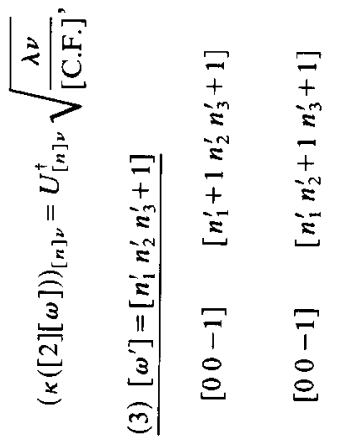




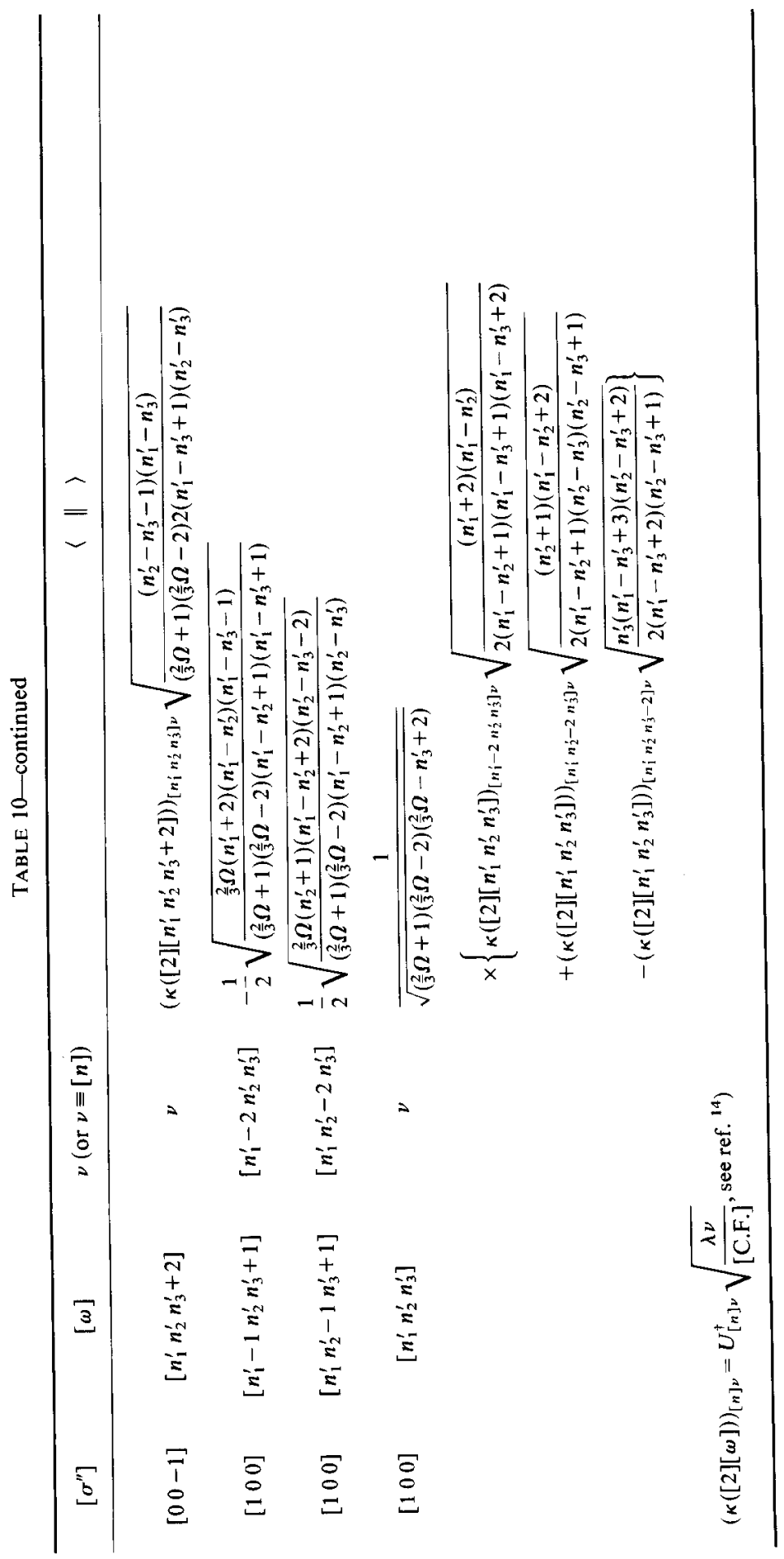


Table 11

$$
\left\langle\left(\frac{\Omega}{3}-1 \frac{\Omega}{3}-1 \frac{\Omega}{3}-1\right)[111][\omega] ;(100)\left[\sigma^{\prime \prime}\right] \|\left(\frac{\Omega}{3} \frac{\Omega}{3}-1 \frac{\Omega}{3}-1\right)[11]\left[\omega^{\prime}\right] \nu^{\prime} \equiv\left[n^{\prime}\right]\right\rangle
$$

(1) $\left[\omega^{\prime}\right]=\left[n_{1}^{\prime}+1 \quad n_{2}^{\prime}+1 n_{3}^{\prime}\right]$

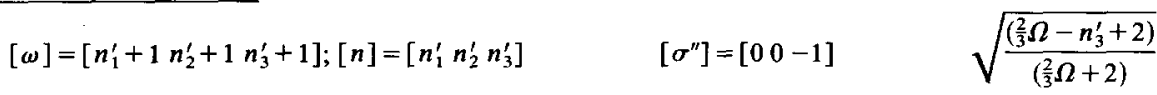

$$
\begin{aligned}
& {[\omega]=\left[n_{1}^{\prime}+1 n_{2}^{\prime}+1 n_{3}^{\prime}-1\right] ;[n]=\left[\begin{array}{lll}
n_{1}^{\prime} & n_{2}^{\prime} & n_{3}^{\prime}-2
\end{array}\right] \quad\left[\sigma^{\prime \prime}\right]=\left[\begin{array}{lll}
1 & 0 & 0
\end{array}\right] \quad-\sqrt{\frac{n_{3}^{\prime}}{\left(\frac{2}{3} \Omega+2\right)}}}
\end{aligned}
$$

(2) $\left[\omega^{\prime}\right]=\left[n_{1}^{\prime}+1 n_{2}^{\prime} n_{3}^{\prime}+1\right]$

$$
\begin{aligned}
& {[\omega]=\left[n_{1}^{\prime}+1 n_{2}^{\prime}+1 n_{3}^{\prime}+1\right] ;[n]=\left[\begin{array}{ll}
n_{1}^{\prime} n_{2}^{\prime} n_{3}^{\prime}
\end{array}\right] \quad\left[\sigma^{\prime \prime}\right]=\left[\begin{array}{lll}
0 & 0-1] & -\sqrt{\frac{\left(\frac{2}{3} \Omega-n_{2}^{\prime}+1\right)}{\left(\frac{2}{3} \Omega+2\right)}}
\end{array}\right.} \\
& {[\omega]=\left[n_{1}^{\prime}+1 n_{2}^{\prime}-1 n_{3}^{\prime}+1\right] ;[n]=\left[\begin{array}{lll}
n_{1}^{\prime} n_{2}^{\prime}-2 n_{3}^{\prime}
\end{array}\right] \quad\left[\sigma^{\prime \prime}\right]=\left[\begin{array}{lll}
1 & 0 & 0
\end{array}\right] \quad-\sqrt{\frac{\left(n_{2}^{\prime}+1\right)}{\left(\frac{2}{3} \Omega+2\right)}}}
\end{aligned}
$$

(3) $\left[\omega^{\prime}\right]=\left[n_{1}^{\prime} n_{2}^{\prime}+1 n_{3}^{\prime}+1\right]$

$$
\begin{array}{lll}
{[\omega]=\left[n_{1}^{\prime}+1 n_{2}^{\prime}+1 n_{3}^{\prime}+1\right] ;[n]=\left[n_{1}^{\prime} n_{2}^{\prime} n_{3}^{\prime}\right]} & {\left[\sigma^{\prime \prime}\right]=\left[\begin{array}{lll}
0 & 0 & -1
\end{array}\right]} & \sqrt{\frac{\left(\frac{2}{3} \Omega-n_{1}^{\prime}\right)}{\left(\frac{2}{3} \Omega+2\right)}} \\
\left.[\omega]=n_{1}^{\prime}-1 n_{2}^{\prime}+1 n_{3}^{\prime}+1\right] ;[n]=\left[n_{1}^{\prime}-2 n_{2}^{\prime} n_{3}^{\prime}\right] & {\left[\sigma^{\prime \prime}\right]=\left[\begin{array}{lll}
1 & 0 & 0
\end{array}\right]} & -\sqrt{\frac{\left(n_{1}^{\prime}+2\right)}{\left(\frac{2}{3} \Omega+2\right)}}
\end{array}
$$

Matrix elements for the case $u^{\prime}=u$ are somewhat more complicated. Their derivation will be illustrated in detail by the matrix element of the operator $A\left(i i^{\prime}\right)$. A new feature arises, since the left action of the intrinsic operator $A\left(i i^{\prime}\right)$ on the intrinsic state $\left[\sigma^{\prime}\right]$ will now connect this to a right state which includes a $z$-space excitation (see entry 4 of table 3 ). Additional recoupling is therefore required to separate the full matrix element into intrinsic and $z$-space parts.

The master equation (24) converts the matrix element of $\boldsymbol{A}\left(\boldsymbol{i i}^{\prime}\right)$ into

$$
\left(\left[\left[n^{\prime}\right] \times\left[\sigma^{\prime}\right]\right]\left[\omega^{\prime}\right] \rho^{\prime} ; I^{\prime}\left\|\mathbb{A}\left(i i^{\prime}\right)_{I_{p}}^{\left(\tilde{\omega}_{p}\right)}\right\|[[n] \times[\sigma]][\omega] \rho ; I\right) .
$$

For $u^{\prime}=u$ the intrinsic operator $\mathbb{A}\left(i i^{\prime}\right)$ must now be worked through to the left to act on the state $\left[\sigma^{\prime}\right]$. For this purpose it will be useful to express the reduced matrix element of a tensor operator $T^{\left[\omega_{0}\right] r_{0}}$ not through its right-coupled form as in eq. (25) but through a left-coupled form. For multiplicity-free cases $[\omega] \times\left[\omega_{0}\right] \rightarrow\left[\omega^{\prime}\right]$

$$
\begin{aligned}
& \left\langle\left[\omega^{\prime}\right] ; I^{\prime}\left\|T^{\left[\omega_{0}\right] I_{0}}\right\|[\omega[\omega]\right. \\
& \quad=\sqrt{\frac{\operatorname{dim}[\omega]}{\operatorname{dim}\left[\omega^{\prime}\right]} \frac{(2 I+1)}{\left(2 I^{\prime}+1\right)}}\left\langle\left[T^{\left[\tilde{\omega}_{0}\right] I_{0}} \times\left[\omega^{\prime}\right] ; I^{\prime}\right]_{\alpha_{\omega} M_{1}}^{[\omega] I} \mid[\omega] \alpha_{\omega} ; I M_{l}\right\rangle,
\end{aligned}
$$


where we have used the symmetry property

$$
\begin{aligned}
& \left\langle[\omega] \alpha_{\omega} ;\left[\omega_{0}\right] \alpha_{0} \mid\left[\omega^{\prime}\right] \alpha_{\omega}^{\prime}\right\rangle \\
& \quad=\sqrt{\frac{\operatorname{dim}\left[\omega^{\prime}\right]}{\operatorname{dim}[\omega]}}\left\langle\left[\omega^{\prime}\right] \alpha_{\omega^{\prime}} ;\left[\tilde{\omega}_{0}\right] \tilde{\alpha}_{0} \mid[\omega] \alpha_{\omega}\right\rangle(-1)^{\hat{\omega}+\hat{\omega}_{0}-\hat{\omega}^{\prime}-\chi\left(\omega_{0}, \alpha_{0}\right)},
\end{aligned}
$$

together with the analogue for the $i$-space angular momentum Wigner coefficient, the hermitian conjugation of the operator $T_{\alpha_{0} M I_{0}}^{\left[\omega_{0}\right] I_{0}}$ via eq. (8), and finally a change in the coupling order of the multiplicity-free product $\left[\omega^{\prime}\right] \times\left[\tilde{\omega}_{0}\right] \rightarrow[\omega]$ which eliminates the phase factor $(-1)^{\hat{\omega}+\hat{\omega}_{0}-\hat{\omega}^{\prime}}$. Note that the coupling order in eq. (47) is a left to right one which is indicated specifically by the arrow, since it is contrary to the standard right to left order which is to apply when not otherwise indicated by an arrow. Since $\left(\tilde{\omega}_{p}\right)=(02)$ or (10) leads to multiplicity-free couplings, eq. (47) can be applied to convert $(46)$ to

$$
\begin{aligned}
& \left(\left[\left[n^{\prime}\right] \times\left[\sigma^{\prime}\right]\right]\left[\omega^{\prime}\right] \rho^{\prime} ; I^{\prime}\left\|\mathbb{A}\left(i i^{\prime}\right)_{I_{p}}^{\left(\tilde{\omega}_{p}\right)}\right\|[[[n] \times[\sigma]][\omega] \rho ; I)\right.
\end{aligned}
$$

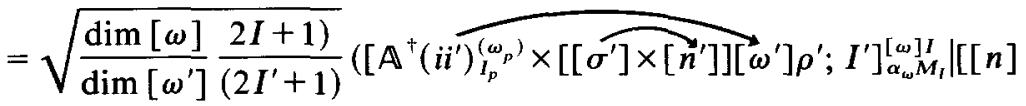

$$
\begin{aligned}
& \left.\times[\sigma]][\omega] \rho \alpha_{\omega} ; I M_{I}\right) \\
& =\sqrt{\frac{\operatorname{dim}[\omega]}{\operatorname{dim}\left[\omega^{\prime}\right]} \frac{(2 I+1)}{\left(2 I^{\prime}+1\right)}} \sum_{[\bar{\sigma}] \bar{\rho}} U\left(\left[\omega_{p}\right]\left[\sigma^{\prime}\right][\omega]\left[n^{\prime}\right] ;[\bar{\sigma}]_{-} \bar{\rho} ;\left[\omega^{\prime}\right] \rho_{-}^{\prime}\right) \\
& \times\left(\left[\left[A^{\dagger}\left(\widehat{\left.i i^{\prime}\right)_{l_{p}}^{\left(\omega_{p}\right)} \times\left[\bar{\sigma}^{\prime}\right.}\right] ; I^{\prime}\right]\left[\widehat{\bar{\sigma}] \times\left[n^{\prime}\right]}\right]_{\alpha_{\omega} ; M_{l}}^{[\omega] \bar{p} ; l} \mid[[n] \times[\sigma]][\omega] \rho \alpha_{\omega} ; I M_{l}\right) .\right.
\end{aligned}
$$

Now we can use the result of entry 4 of table 3 to note that

$$
\begin{aligned}
\left(\left[\mathbb{A}^{\dagger}\right.\right. & \left.\left(i i^{\prime}\right)_{I_{p}}^{\left(\omega_{p}\right)} \times\left[\sigma^{\prime}\right] ; I^{\prime}\right]_{\bar{\alpha} ; M_{l}}^{[\bar{\sigma}] ; I} \mid \\
= & \left(\left[\mathcal{A}^{\dagger}\left(i i^{\prime}\right)_{i_{p}}^{\left(\omega_{p}\right)} \times\left[\sigma^{\prime}\right] ; I^{\prime}\right]_{\bar{\alpha} ; M_{I}}^{[\bar{\sigma}]] I} \mid[[2] \times[\sigma]][\bar{\sigma}] \bar{\alpha} ; I M_{I}\right) \times\left([[2] \times[\sigma]][\bar{\sigma}] \bar{\alpha} ; I M_{l} \mid\right. \\
= & \sqrt{\frac{\operatorname{dim}\left[\sigma^{\prime}\right]}{\operatorname{dim}[\bar{\sigma}]} \frac{\left(2 I^{\prime}+1\right)}{(2 I+1)}}\left(\left[\sigma^{\prime}\right] ; I^{\prime}\left\|\mathbb{A}\left(i i^{\prime}\right)_{I_{p}}^{\left(\tilde{\omega}_{p}\right)}\right\| \|[[2] \times[\sigma]][\bar{\sigma}] ; I\right) \\
& \times\left([[2] \times[\sigma]][\bar{\sigma}] \bar{\alpha} ; I M_{I} \mid .\right.
\end{aligned}
$$

Eqs. (49) and (50) lead to

$$
\begin{aligned}
\left(\left[\left[n^{\prime}\right] \times\left[\sigma^{\prime}\right]\right]\left[\omega^{\prime}\right] \rho^{\prime} ; I^{\prime}\left\|\mathbb{A}\left(i i^{\prime}\right) i_{p}^{\left(\tilde{\omega}_{p}\right)}\right\|[[[n] \times[\sigma]][\omega] \rho ; I)\right. \\
=\sum_{[\bar{\sigma}] \bar{\rho}} \sqrt{\frac{\operatorname{dim}[\omega]}{\operatorname{dim}\left[\omega^{\prime}\right]} \frac{\operatorname{dim}\left[\bar{\sigma}^{\prime}\right]}{\operatorname{sim}[\bar{\sigma}]}} U\left(\left[\omega_{p}\right]\left[\sigma^{\prime}\right][\omega]\left[n^{\prime}\right] ;[\bar{\sigma}]-\bar{\rho} ;\left[\omega^{\prime}\right] \rho_{-}^{\prime}\right) \\
\quad \times\left(\left[\left[n^{\prime}\right] \times[[2] \times[\sigma]][\bar{\sigma}]\right][\omega] \bar{\rho} \alpha_{\omega} ; I M_{I} \mid[[n] \times[\sigma]][\omega] \rho \alpha_{\omega} ; I M_{I}\right) \\
\quad \times\left(\left[\sigma^{\prime}\right] ; I^{\prime}\left\|\mathbb{A}\left(i i^{\prime}\right)_{I_{p}}^{\left(\tilde{\omega}_{p}\right)}\right\|[[[2] \times[\sigma]][\bar{\sigma}] ; I)\right.
\end{aligned}
$$




$$
\begin{aligned}
= & \sum_{[\bar{\sigma}] \bar{\rho}} \sqrt{\frac{\operatorname{dim}[\omega]}{\operatorname{dim}\left[\omega^{\prime}\right]} \frac{\operatorname{dim}\left[\sigma^{\prime}\right]}{\operatorname{dim}[\bar{\sigma}]}} U\left(\left[\omega_{p}\right]\left[\sigma^{\prime}\right][\omega]\left[n^{\prime}\right] ;[\bar{\sigma}]_{-} \bar{\rho} ;\left[\omega^{\prime}\right] \rho_{-}^{\prime}\right) \\
& \times U\left([\sigma][2][\omega]\left[n^{\prime}\right] ;[\bar{\sigma}]_{-} \bar{\rho} ;[n]_{-} \rho\right) \\
& \times\left([n]\|z\|\left[n^{\prime}\right]\right)\left(\left[\sigma^{\prime}\right] ; I^{\prime}\left\|\mathbb{A}\left(i i^{\prime}\right)_{I_{p}}^{\left(\tilde{\omega}_{p}\right)}\right\| \|[[2] \times[\sigma]][\bar{\sigma}] ; I\right) .
\end{aligned}
$$

This result is tabulated as entry 6 in table 4.

Finally, the matrix element of $P\left(i i^{\prime}\right)_{I_{p}}^{(p)\left(\omega_{0}\right)}$ follows from

$$
\begin{aligned}
\left(\left[\left[n^{\prime}\right] \times\left[\sigma^{\prime}\right]\right]\left[\omega^{\prime}\right] \rho^{\prime} ; I^{\prime}\left\|\Gamma\left(P\left(i i^{\prime}\right){ }_{I_{p}}^{(p)\left(\omega_{0}\right)}\right)\right\|[[n] \times[\sigma]][\omega] \rho ; I\right)_{\rho_{0}} \\
=\left(\left[\left[n^{\prime}\right] \times\left[\sigma^{\prime}\right]\right]\left[\omega^{\prime}\right] \rho^{\prime} ; I^{\prime}\left\|\mathbb{P}\left(i i^{\prime}\right){ }_{I_{p}}^{(p)\left(\omega_{0}\right)}\right\|[[[n] \times[\sigma]][\omega] \rho ; I)_{\rho_{0}}\right. \\
\quad+c_{\omega_{0}}^{(p)}\left(\left[\left[n^{\prime}\right] \times\left[\sigma^{\prime}\right]\right]\left[\omega^{\prime}\right] \rho^{\prime} ; I^{\prime}\left\|\left[\mathbb{A}\left(i i^{\prime}\right)_{I_{p}}^{\left[\tilde{\omega}_{p}\right]} \times Z^{[2]}(z)\right]^{\left[\omega_{0}\right]}\right\|[[n] \times[\sigma]][\omega] \rho ; I\right)_{\rho_{0}} .
\end{aligned}
$$

Using (33b), the second term can be reexpressed as

$$
\begin{aligned}
\left(\left[\left[n^{\prime}\right] \times\left[\sigma^{\prime}\right]\right]\left[\omega^{\prime}\right] \rho^{\prime} ; I^{\prime}\left\|\left(\mathbb{A}\left(i i^{\prime}\right)_{I_{p}}^{\left[\tilde{\omega}_{p}\right]} \times Z^{[2]}\right]^{\left[\omega_{0}\right]}\right\|[[n] \times[\sigma]][\omega] \rho ; I\right)_{\rho_{0}} \\
=\sum_{\left[\omega^{\prime \prime}\right]\left[n^{\prime \prime}\right] \rho^{\prime \prime}} U\left([\omega][2]\left[\omega^{\prime}\right]\left[\tilde{\omega}_{p}\right] ;\left[\omega^{\prime \prime}\right]_{--} ;\left[\omega_{0}\right]_{-} \rho_{0}\right) \\
\quad \times U\left([\sigma][n]\left[\omega^{\prime \prime}\right][2] ;[\omega] \rho_{-} ;\left[n^{\prime \prime}\right]_{-} \rho^{\prime \prime}\right)\left(\left[n^{\prime \prime}\right]\|z\|[n]\right) \\
\quad \times\left(\left[\left[n^{\prime}\right] \times\left[\sigma^{\prime}\right]\right]\left[\omega^{\prime}\right] \rho^{\prime} ; I^{\prime}\left\|\mathbb{A}\left(i i^{\prime}\right)_{I_{p}}^{\left[\tilde{\omega}_{p}\right]}\right\|\left[\left[n^{\prime \prime}\right] \times[\sigma]\right]\left[\omega^{\prime \prime}\right] \rho^{\prime \prime} ; I\right)_{\rho_{0}} .
\end{aligned}
$$

The matrix element of $A\left(i i^{\prime}\right)$ follows from eq. (51). The full result for the matrix element of $P\left(i i^{\prime}\right)_{t_{p}}^{(p)\left(\omega_{0}\right)}$ is given as entry 7 in table 4 . Note that the first term of eq. (52) leads to 9-j type SU(3) recoupling coefficient with one [0]. In the cases where multiplicity labels $\rho_{\sigma}$ and $\rho_{0}$ are not needed this could be converted to a U-coefficient as in entry 3 . In the most general case, however, it is best left in the $9-j$ type form since some needed $1 \leftrightarrow 2 \mathrm{SU}(3)$ reordering transformations are then no longer simple.

Entry 7 completes the list of needed reduced matrix elements for the one-body operators and pair creation and annihilation operators. Matrix elements for states with $u^{\prime}=u+2$ can be obtained from entries 3-5 via hermitian conjugation. Similarly, matrix elements of pair creation operators connecting states with $u^{\prime}=u$ can be obtained from entry 6 of table 4 via

$$
\begin{aligned}
\left\langle\left[\sigma^{\prime}\right]\left[\omega^{\prime}\right] \nu^{\prime} ; I^{\prime}\left\|A^{\dagger}\left(i i^{\prime}\right)_{i_{p}}^{\left(\omega_{p}\right)}\right\|[\sigma][\omega] \nu ; I\right\rangle \\
=(-1)^{1+I+I_{p}-I^{\prime}+\hat{\omega}+\hat{\omega}_{0}-\hat{\omega}^{\prime}} \sqrt{\frac{(2 I+1) \operatorname{dim}[\omega]}{\left(2 I^{\prime}+1\right) \operatorname{dim}\left[\omega^{\prime}\right]}} \\
\quad \times\left\langle[\sigma][\omega] \nu ; I\left\|A\left(i i^{\prime}\right)_{I_{p}}^{\left(\bar{\omega}_{p}\right)}\right\|\left[\sigma^{\prime}\right]\left[\omega^{\prime}\right] \nu^{\prime} ; I^{\prime}\right\rangle .
\end{aligned}
$$

The final results as catalogued in table 4 give the needed matrix elements in terms of, (1), readily available ${ }^{18}$ ) SU(3) recoupling coefficients, (2), the $K$-matrix elements of VCS theory which can be read from ref. ${ }^{14}$ ) or calculated by the techniques of 
ref. ${ }^{14}$ ), and, finally, (3), a few starting matrix elements of simple shell-model type. Examples of the latter are given for states of low heritage, $u$, in the appendix. In cases of greater $u$ they can be calculated by standard cfp techniques. Table 4 includes all possible SU(3) multiplicity labels. For states with $u \leqslant 2$ most of these never come into play. The label, $\rho_{0}$, which is part of the $k$-space $\mathrm{SU}(3), i$-space $\mathrm{SU}(2)$ reduced matrix element, is needed only for entries 4 and 7 and the special case $\left(\omega_{0}\right)=$ (11), $\left(\omega^{\prime}\right)=(\omega)$. Note that the $\rho_{0}$ dependence arises entirely from the SU(3)-recoupling coefficients in the expressions for the reduced matrix elements. In the codes of ref. ${ }^{18}$ ) these are matched by the $\rho_{0}$ dependence in the $\mathrm{SU}(3) \supset \mathrm{SO}(3)$ reduced Wigner coefficients which are needed to construct the full matrix elements via eq. (26).

Although some of the entries of table 4 involve a number of SU(3) Racah coefficients and summations over $U(3)$ quantum numbers, these coefficients can be given in analytic form in certain very simple cases and the sums can be performed to lead to fairly general analytic formulae. E.g., for $\left[\omega_{0}\right]=[0]$ and $u=u^{\prime}=1$ so that $[\sigma]=[1]$, entry 7 of table 4 , together with entries 4 and 6 of table 3 , lead to the simple results, (valid for $I_{p} \neq 0$ ),

$$
\left\langle[[1] \times[n]][\omega] ; i\left\|P\left(i i^{\prime}\right)_{\}_{p}^{(s)}}^{(0]}\right\|[[1] \times[n]][\omega] ; i^{\prime}\right\rangle
$$

(1) for $[\omega]=\left[n_{1}+1 n_{2} n_{3}\right]$ :

$$
=\frac{\left(1+\delta_{i i}\right)}{2} \sqrt{\frac{\left(2 I_{p}+1\right)}{3(2 i+1)}}\left\{\frac{\left(\frac{2}{3} \Omega-2\right)\left(\frac{2}{3} \Omega+1\right)-2 n_{1}\left(\frac{2}{3} \Omega+1\right)-2\left(n_{2}+n_{3}\right)}{\left(\frac{2}{3} \Omega-2\right)\left(\frac{2}{3} \Omega+1\right)}\right\},
$$

(2) for $[\omega]=\left[n_{1} n_{2}+1 n_{3}\right]$ :

$$
=\frac{\left(1+\delta_{i i^{\prime}}\right)}{2} \sqrt{\frac{\left(2 I_{p}+1\right)}{3(2 i+1)}}\left\{\frac{\left(\frac{2}{3} \Omega\right)^{2}+\left(\frac{2}{3} \Omega\right)-2-2 n_{2}\left(\frac{2}{3} \Omega+1\right)-2\left(n_{1}+n_{3}\right)}{\left(\frac{2}{3} \Omega-2\right)\left(\frac{2}{3} \Omega+1\right)}\right\},
$$

(3) for $[\omega]=\left[n_{1} n_{2} n_{3}+1\right]$ :

$$
=\frac{\left(1+\delta_{i i^{\prime}}\right)}{2} \sqrt{\frac{\left(2 I_{p}+1\right)}{3(2 i+1)}}\left\{\frac{\left(\frac{2}{3} \Omega\right)^{2}+3\left(\frac{2}{3} \Omega\right)-2-2 n_{3}\left(\frac{2}{3} \Omega+1\right)-2\left(n_{1}+n_{2}\right)}{\left(\frac{2}{3} \Omega-2\right)\left(\frac{2}{3} \Omega+1\right)}\right\},
$$

where we have used the simple starting matrix elements of $P\left(\dddot{i i}^{\prime}\right)_{I_{p}}^{(\mathrm{s})\left[\omega_{0}\right]}$ from the appendix.

With $I_{p}=0$, the operator

$$
\sum_{i} \sqrt{3(2 i+1)} P(i i)_{i_{p}=0}^{(\mathrm{s})(00)}=N_{\mathrm{op}} .
$$

is the simple number operator. In this case, entry 7 of table 4 together with entries 4 and 6 of table 3 verify that the diagonal matrix element of this operator is simply the total number of particles, $N=\sigma_{1}+\sigma_{2}+\sigma_{3}+n_{1}+n_{2}+n_{3}$. Similarly, the operators

$$
\sum_{i} \sqrt{(2 i+1)} P(i i)_{K M_{K}, I_{p}=0}^{(s)(11)}=C_{K M_{K}}^{(11)}
$$

are the SU(3) generators. Although their matrix elements follow at once from the general theory of generator matrix elements of ref. ${ }^{14}$ ), entry 7 of table 4 verifies 
that the reduced matrix elements of this operator are given in terms of the SU(3) Casimir invariant by

$$
\begin{gathered}
\left\langle[\sigma][\omega] \nu ; I \|\left|\sum_{i} \sqrt{(2 i+1)} P(i i)_{I_{p}=0}^{(\mathrm{s})(11)}\right|||[\sigma][\omega] \nu ; I\right\rangle_{\rho_{0}} \\
=\delta_{\rho_{0} 1} \sqrt{\frac{2}{3}\left[\lambda_{\omega}^{2}+\mu_{\omega}^{2}+\lambda_{\omega} \mu_{\omega}+3\left(\lambda_{\omega}+\mu_{\omega}\right)\right]} .
\end{gathered}
$$

Note that only the $\rho_{0}=1$ matrix element survives for this $\mathrm{SU}(3)$ generator.

It should be noted, in particular, that the $z$-space realization of the SU(3) generator, $\sum_{i} \sqrt{(2 i+1)} \Gamma\left(P(i i)_{\alpha, I_{p}=0}^{(\mathrm{s})\left(\omega_{0}\right)}\right)$, as given by eq. $(12 \mathrm{~d})$ is quite different from the "standard" $z$-space realization as given by eq. (12) of ref. ${ }^{14}$ ). The latter involves $z$-derivative operators. It is well known that coherent state realizations of operators are not unique due to the overcompleteness of coherent states. It is, however, gratifying to note that two quite different realizations, $\Gamma(\boldsymbol{O})$, for the same operator lead to the same final matrix elements. In similar fashion, the operator

$$
\sum_{i} \sqrt{\frac{3}{2}(2 i+1)} A(i i)_{J M, I=0}^{(02)}
$$

is the pair annihilation generator of $\mathrm{Sp}(6)$ whose matrix elements follow most simply from eqs. (31) and (29) of ref. ${ }^{14}$ ) through its simple $z$-derivative realization, eq. (12) of ref. ${ }^{14}$ ). Entry 3 of table 4 again verifies this simple result, demonstrating again that two quite different realizations, $\Gamma(\boldsymbol{O})$, of the same operator lead to the same matrix elements.

Another interesting special case involves the states with $u=2,[\sigma]=[2]$ and $[\omega]=[n 00]$ where $n$ is the total particle number, (an even number), i.e., states of highest possible $S U(3)$ symmetry. In this case entry 7 of table 4 leads to

$$
\begin{aligned}
\langle[2][\omega] & \left.=[n 00] ;[i \times i] I^{\prime}\left\|P(i i)_{I_{p}}^{(s)\left[\omega_{0}\right]}\right\|[2][\omega]=[n 00] ;[i \times i] I\right\rangle \\
& =\left[\frac{\left(\frac{2}{3} \Omega-2 n\right)}{\left(\frac{3}{2} \Omega-4\right)}\right] 2 \sqrt{\frac{(2 I+1)}{3(2 i+1)}} U\left(I i I^{\prime} i ; i I_{p}\right) F_{\left[\omega_{0}\right]},
\end{aligned}
$$

$$
\text { with } F_{(00)}=1, \quad F_{(11)}=\sqrt{2(n+3) / n} \text {. }
$$

where we have used the starting reduced matrix element of $P(i i)_{I_{p}}^{(s)\left[\omega_{0}\right]}$ between states of type [2]; $i \times i] I$ from the appendix. Other cases with different combinations of $i, i^{\prime}$ with $i \neq i^{\prime}$ follow from the analogous reduced matrix elements of the appendix.

Another important matrix element for these simple $u=2$ states is the $u$-breaking matrix element with $u^{\prime}=0$. Entry 4 of table 4 now leads to the simple value

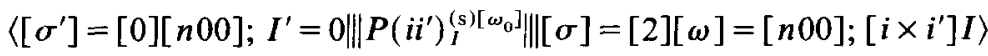

$$
\begin{aligned}
& =\sqrt{2\left(1+\delta_{i i^{\prime}}\right)} \sqrt{(2 I+1)} \sqrt{\frac{\left(\frac{2}{3} \Omega-n\right)}{\left(\frac{2}{3} \Omega\right)\left(\frac{2}{3} \Omega-2\right)}} f_{\left[\omega_{0}\right]} \text {, } \\
& \text { with } f_{(00)}=\sqrt{\frac{1}{6} n}, \quad f_{(11)}=\sqrt{\frac{8}{15}(n+3)} \text {. }
\end{aligned}
$$

Eqs. (60) and (61) have been derived by Ginocchio ${ }^{19}$ ). 


\section{Summary}

Very general expressions have been given for the matrix elements in the $\operatorname{Sp}(6) \supset$ $U(3)$ basis of the fermion dynamic symmetry model of single-nucleon creation and annihilation operators, of the most general one-body operators, and of the pair creation and annihilation operators. These expressions reduce the general matrix elements for arbitrary nucleon number, $n$, and arbitrary $U(3)$ representation, to those for nucleon numbers $u$ and $u^{\prime}$ where these are the S, D-pair seniority numbers which come into play. Effectively, an $n$-particle calculation has therefore been reduced to one involving $u$ (or $u^{\prime}$ ) particles. The matrix elements depend only on readily available $S U(3)$ recoupling coefficients ${ }^{18}$ ) and on the $K$-matrix elements of VCS theory. For states with $u \leqslant 2$ the latter have been given in general analytic form ${ }^{14}$ ). If the low-lying states in real nuclei are dominated by states of low heritage it becomes feasible to evaluate nuclear matrix elements of the most general operators, with $i$-space spins different from zero. An earlier study by Halse ${ }^{20}$ ) in the sd shell has shown that the $u=0$ states have very little overlap with the eigenfunctions of a realistic shell-model hamiltonian, so that the validity of the low heritage model is open to serious question, at least for very light nuclei. With the techniques of this investigation it should now be feasible to make an honest test of the validity of the fermion dynamic symmetry model in rotational nuclei in the actinide region, where the $\mathrm{Sp}(6) \supset \mathrm{U}(3)$ branch of the model may have some applicability.

\section{Appendix}

\section{STARTING MATRIX ELEMENTS}

The simple starting matrix elements connecting states of heritage, $u$, to states of heritage $u^{\prime}=u$, or $u \pm 1, u \pm 2$, via the operators $b_{i}, b_{i}^{\dagger}, A\left(i i^{\prime}\right)$, or $P\left(i i^{\prime}\right)$ can be calculated by standard shell-model techniques. For $u\left(u^{\prime}\right) \leqslant 2$ the results are very simple. For $u\left(u^{\prime}\right) \geqslant 3$, standard cfp techniques can be used. For $u\left(u^{\prime}\right) \leqslant 2$ the single-nucleon creation (annihilation) operator matrix elements are

$$
\begin{aligned}
\left\langle[1] ; i\left\|b_{i}^{\dagger}\right\|[0] ; 0\right\rangle & =1, \\
\left\langle[0] ; 0\|\| \tilde{b}_{i} \|[1] ; i\right\rangle & =\sqrt{3(2 i+1)}, \\
\left\langle\left[\sigma^{\prime}\right] ;\left[i \times i^{\prime}\right] I^{\prime}\left\|b_{i^{\prime}}^{\dagger}\right\|[1] ; i\right\rangle & =-\sqrt{\left(1+\delta_{i i^{\prime}}\right)}, \\
\left\langle\left[\sigma^{\prime}\right] ;\left[i \times i^{\prime}\right] I^{\prime}\left\|b_{i}^{\dagger}\right\|[1] ; i^{\prime}\right\rangle & =\sqrt{\left(1+\delta_{i i^{\prime}}\right)}(-1)^{i+i^{\prime}-I^{\prime}+\hat{\sigma}^{\prime}}, \\
\left\langle[1] ; i^{\prime}\left\|\tilde{b}_{i}\right\|[\sigma] ;\left[i \times i^{\prime}\right] I\right\rangle & =\sqrt{\left(1+\delta_{i i^{\prime}}\right)} \sqrt{\frac{\operatorname{dim}[\sigma]}{3} \frac{(2 I+1)}{\left(2 i^{\prime}+1\right)}}, \\
\left\langle[1] ; i\left\|\tilde{b}_{i^{\prime}}\right\|[\sigma] ;\left[i \times i^{\prime}\right] I\right\rangle & =\sqrt{\left(1+\delta_{i i^{\prime}}\right)} \sqrt{\frac{\operatorname{dim}[\sigma]}{3} \frac{(2 I+1)}{(2 i+1)}(-1)^{i+i^{\prime}-I+1+\hat{\sigma}},}
\end{aligned}
$$

with $[\sigma]$ or $\left[\sigma^{\prime}\right]=[2]$ or $[11]$. 
Matrix elements of $A\left(i i^{\prime}\right)$ and $P\left(i i^{\prime}\right)$ can be obtained from these via intermediate state sums which lead to the relations (valid for general $[\sigma]$ and $\left[\sigma^{\prime}\right]$ ).

$\left\langle\left[\sigma^{\prime}\right] ; I^{\prime} \| \boldsymbol{A}(\ddot{i})_{I_{p}}^{\left.\left[\tilde{\omega}_{p}\right] \|[\sigma] ; I\right\rangle}\right.$

$$
\begin{aligned}
= & \sum_{\left[\sigma^{\prime \prime}\right]} \sum_{I^{\prime \prime}} U\left([\sigma][11]\left[\sigma^{\prime}\right][11] ;\left[\sigma^{\prime \prime}\right]\left[\tilde{\omega}_{p}\right]\right) U\left(I^{\prime} I^{\prime} i ; I^{\prime \prime} I_{p}\right) \\
& \times(-1)^{i+i^{\prime}-I_{p}+\hat{\omega}_{p}}\left\langle\left[\sigma^{\prime}\right] ; I^{\prime}\left\|\tilde{b}_{i}^{[11]}\right\|\left[\mid \sigma^{\prime \prime}\right] ; I^{\prime \prime}\right\rangle\left\langle\left[\sigma^{\prime \prime}\right] ; I^{\prime \prime}\left\|\tilde{b}_{i^{\prime}}^{[1]}\right\|[\sigma] ; I\right\rangle
\end{aligned}
$$

and

$\left\langle\left[\sigma^{\prime}\right] ; I^{\prime}\left\|\left[b_{i}^{\dagger} \times \tilde{b}_{i^{\prime}}\right]_{I_{0}}^{\left[\omega_{0}\right]}\right\|[\sigma] ; I\right\rangle_{\rho_{0}}$

$$
\begin{aligned}
& =\sum_{\left[\sigma^{\prime \prime}\right]} \sum_{I^{\prime \prime}} U\left([\sigma][11]\left[\sigma^{\prime}\right][1] ;\left[\sigma^{\prime \prime}\right]_{--} ;\left[\omega_{0}\right]_{-} \rho_{0}\right) U\left(I i^{\prime} I^{\prime} i ; I^{\prime \prime} I_{0}\right) \text {, } \\
& \times(-1)^{i+i^{\prime}-I_{0}+\hat{\omega}_{0}}\left\langle\left[\sigma^{\prime}\right] ; I^{\prime}\left\|b_{i}^{\dagger[1]}\right\|\left[\sigma^{\prime \prime}\right] ; I^{\prime \prime}\right\rangle\left\langle\left[\sigma^{\prime \prime}\right] ; I^{\prime \prime}\left\|b_{i^{\prime}}^{[11]}\right\|[\mid \sigma] ; I\right\rangle .
\end{aligned}
$$

Interesting special cases include

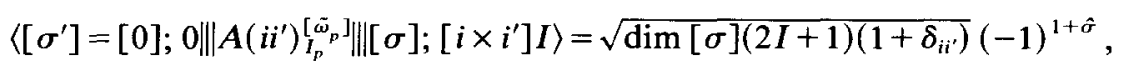

with $[\sigma]=[2],[11]$;

$$
\begin{aligned}
\left\langle\left[\sigma^{\prime}\right]\right. & \left.=[1] ; i \| P\left(i i^{\prime}\right) I_{p}^{(p)\left(\omega_{0}\right) \|[\sigma]}=[1] ; i^{\prime}\right\rangle \\
& =\frac{1}{2} \sqrt{\frac{\left(2 I_{p}+1\right) \operatorname{dim}\left(\omega_{0}\right)}{3(2 i+1)}}\left(1+\delta_{i i^{\prime}}\right), \\
\left\langle\left[\sigma^{\prime}\right]\right. & \left.=[1] ; i^{\prime} \| P\left(i i^{\prime}\right) I_{p}^{(p)\left(\omega_{0}\right) \|[[\sigma]}=[1] ; i\right\rangle \\
& =\mp \frac{1}{2} \sqrt{\frac{\left(2 I_{p}+1\right) \operatorname{dim}\left(\omega_{0}\right)}{3\left(2 i^{\prime}+1\right)}}(-1)^{i+i^{\prime}-I_{p}}\left(1+\delta_{i i^{\prime}}\right),
\end{aligned}
$$

with upper [or lower] sign for $(p)=(\mathrm{s})[$ or $(p)=(\mathrm{a})$ ].

$\left\langle[2] ;[i \times i] I^{\prime}\left\|P(i i)_{I_{p}}^{(\mathrm{s})\left(\omega_{0}\right)}\right\|[[2][i \times i] I\rangle\right.$

$$
=2 \sqrt{\frac{(2 I+1) f\left(\omega_{0}\right)}{3(2 i+1)}} U\left(I I^{\prime} i ; i I_{p}\right),
$$

$\left\langle[2] ;[i \times i] I^{\prime} \mid\left\|P\left(i i^{\prime}\right)_{I_{p}}^{(\mathrm{s})\left(\omega_{0}\right)}\right\|[2]\left[i \times i^{\prime}\right] I\right\rangle$

$$
=\sqrt{\frac{(2 I+1) f\left(\omega_{0}\right)}{6(2 i+1)}}(-1)^{I_{p}-I} U\left(I i^{\prime} I^{\prime} i ; i I_{p}\right),
$$

$\left\langle[2] ;\left[i^{\prime} \times i^{\prime}\right] I^{\prime}\left\|P\left(i i^{\prime}\right)_{I_{p}}^{(\mathrm{s})\left(\omega_{0}\right)}\right\|[2] ;\left[i \times i^{\prime}\right] I\right\rangle$

$$
=\sqrt{\frac{(2 I+1) f\left(\omega_{0}\right)}{6\left(2 i^{\prime}+1\right)}} U\left(I i I^{\prime} i^{\prime} ; i^{\prime} I_{p}\right),
$$

$\left\langle[2] ;\left[i \times i^{\prime}\right]^{\prime} I^{\prime}\left\|P(i i)_{I_{p}}^{(\mathrm{s})\left(\omega_{0}\right)}\right\|[2] ;\left[i \times i^{\prime}\right] I\right\rangle$

$$
=(-1)^{i-i^{\prime}-I^{\prime}} \sqrt{\frac{(2 I+1) f\left(\omega_{0}\right)}{3\left(2 i^{\prime}+1\right)}} U\left(I i I^{\prime} i ; i^{\prime} I_{p}\right),
$$




$$
\begin{aligned}
\langle[2] ; & {\left.\left[i \times i^{\prime}\right] I^{\prime}\left\|P\left(i i^{\prime}\right)_{I_{p}}^{(s)\left(\omega_{0}\right)}\right\|[2][i \times i] I\right\rangle } \\
& =\sqrt{\frac{(2 I+1) f\left(\omega_{0}\right)}{6(2 i+1)}} U\left(I i I^{\prime} i^{\prime} ; i I_{p}\right),
\end{aligned}
$$

with $f((00))=1$; and $f((11))=5$.

\section{References}

1) J.N. Ginocchio, Ann. of Phys. 126 (1980) 234

2) C.L. Wu, D.J. Feng, X.G. Chen, J.Q. Chen and M.W. Guidry, Phys. Lett. B168 (1986) 313

3) J.Q. Chen, D.H. Feng, and C.L. Wu, Phys. Rev. C34 (1986) 2269

4) C.L. Wu, D.H. Feng, X.G. Chen, J.Q. Chen and M.W. Guidry, Phys. Rev. C36 (1987) 1157

5) Z.M. Lu, X.W. Pan, J.Q. Chen, X.G. Chen and D.H. Feng, Phys. Rev. C37 (1988) 2789

6) X.L. Han, M.W. Guidry, D.H. Feng, K.X. Wang and C.L. Wu, Phys. Lett. B192 (1987) 253

7) H. Wu, C.L. Wu, D.H. Feng and M.W. Guidry, Phys. Rev. C37 (1988) 1739

8) H. Wu, D.H. Feng, C.L. Wu, C.P. Li and M.W. Guidry, Phys. Lett. B193 (1987) 163

9) D.J. Rowe, J. Math. Phys. 25 (1984) 2662

10) D.J. Rowe, R. LeBlanc and K.T. Hecht, J. Math. Phys. 29 (1988) 287

11) K.T. Hecht, The vector coherent state method and its application to problems of higher symmetries, Lecture Notes in Physics 290 (Springer, Berlin, 1987)

12) K.T. Hecht, Nucl. Phys. A444 (1985) 189

13) K.T. Hecht, Nucl. Phys. A475 (1987) 276

14) K.T. Hecht, Nucl. Phys. A484 (1988) 61

15) J.Q. Chen, X.G. Chen, D.H. Feng, C.L. Wu, J.N. Ginocchio and M.W. Guidry, Phys. Rev. C (in press)

16) J.L. Ping, to be published

17) K.T. Hecht, Nucl. Phys. A493 (1989) 29

18) J.P. Draayer and Y. Akiyama, J. Math. Phys. 14 (1973) 1904; Comput. Phys. Comm. 5 (1973) 405

19) J.N. Ginocchio, private communication

20) P. Halse, Phys. Rev. C36 (1987) 372 